\title{
Dark matter from dark photons: A taxonomy of dark matter production
}

\author{
Thomas Hambye, ${ }^{1,2, *}$ Michel H. G. Tytgat $\odot,{ }^{1, \dagger}$ Jérôme Vandecasteele, ${ }^{1, *}$ and Laurent Vanderheyden ${ }^{1, \S}$ \\ ${ }^{1}$ Service de Physique Théorique, Université Libre de Bruxelles, \\ Bld du Triomphe CP225, 1050 Brussels, Belgium \\ ${ }^{2}$ Instituto de Física Teórica, IFT-UAM/CSIC, U.A.M., Cantoblanco, 28049 Madrid, Spain
}

(Received 20 September 2019; published 18 November 2019)

\begin{abstract}
We analyze how dark matter (DM) can be produced in the early Universe, working in the framework of a hidden sector charged under a $U(1)^{\prime}$ gauge symmetry and interacting with the Standard Model through kinetic mixing. Depending on the masses of the dark matter particle and of the dark photon, as well as on the hidden $U(1)^{\prime}$ gauge coupling and the kinetic mixing parameter, we classify all the distinct regimes along which the observed dark matter relic density can be accounted for. We find that nine regimes are potentially operative to produce the DM particles, and these operate along five distinct dynamical mechanisms. Among these, four regimes are new and correspond to regimes in which the DM particles are produced by on-shell dark photons. One of them proceeds along a new dynamical mechanism, which we dub sequential freeze-in. We argue that such regimes and the associated dynamical mechanisms are characteristic of DM models for which, on top of the Standard Model and the dark sector, there are other massive, but relatively light particles — akin to the dark photon - that interact with both the SM and the DM sectors.
\end{abstract}

DOI: 10.1103/PhysRevD.100.095018

\section{INTRODUCTION}

As the nature of dark matter (DM) remains a mystery, it is possible that DM is a particle that belongs to a whole new hidden sector. This hidden sector may be coupled through the Standard Model (visible sector) through a few possible portals [1]. The question we study further in the present work is how to account, in generic terms, for the abundance of a dark matter (DM) particle produced through portals. For the case of the kinetic mixing portal with a massless dark photon, this has been addressed in much detail in Ref. [2]. That work also includes the case of dark matter creation through the Higgs portal. In the present work, we consider the possible impact of a finite dark photon mass. More generically, our study applies to DM production in models in which a relatively light particle couples both to the DM and to SM particles.

The existence of a massive dark photon, associated with a hidden $U(1)^{\prime}$ gauge interaction, has been the object of many investigations, both theoretically and experimentally;

\footnotetext{
*thambye@ulb.ac.be

†tytgat@ulb.ac.be

\#vdecast@ulb.ac.be

lavdheyd@ulb.ac.be
}

Published by the American Physical Society under the terms of the Creative Commons Attribution 4.0 International license. Further distribution of this work must maintain attribution to the author(s) and the published article's title, journal citation, and DOI. Funded by SCOAP. see, e.g., the reviews in Refs. [3-5]. This possibility is well motivated, very rich phenomenologically, and moreover, is directly related to the DM problem. Indeed, a gauge symmetry is a most natural way to stabilize a particle [6-10]. Specifically, we consider the following simple and popular model:

$$
\begin{aligned}
\mathcal{L} \supset & -\frac{1}{4} B^{\prime \mu \nu} B_{\mu \nu}^{\prime}-\frac{\hat{\epsilon}}{2} B^{\mu \nu} B_{\mu \nu}^{\prime}+\frac{1}{2} m_{\gamma^{\prime}}^{2} B^{\prime \mu} B_{\mu}^{\prime} \\
& +i \bar{\chi} \not \supset \chi-m_{\mathrm{DM}} \bar{\chi} \chi+\cdots
\end{aligned}
$$

Here $\chi$ is a Dirac fermion, singlet under the SM gauge group but charged under $U(1)^{\prime}$ and will be our DM candidate. Its covariant derivative is $D_{\mu}=\partial_{\mu}+i e^{\prime} B_{\mu}^{\prime}$, where $B_{\mu}^{\prime}$ and $e^{\prime}$ are the $U(1)^{\prime}$ gauge field and coupling. This dark, or hidden, sector is coupled to the Standard Model (SM) sector through the so-called kinetic mixing portal term, which mixes the dark gauge field with the SM hypercharge one with a mixing parameter $\hat{\epsilon}$ [11]. This Lagrangian thus involves four new parameters: $m_{\mathrm{DM}}, \hat{\epsilon}, e^{\prime}$, and what will turn out to be the mass of the dark photon particle, $m_{\gamma^{\prime}}$. Equivalently, we will make use of the hiddensector fine structure constant $\alpha^{\prime} \equiv e^{2} / 4 \pi$ and of the combination $\kappa=\hat{\epsilon} \cos \theta_{W} e^{\prime} / e$, which is the millicharge of the DM in the limit $m_{\gamma^{\prime}} \rightarrow 0$. The dark photon mass can arise either through the Stuickelberg [12] or through the Brout-Englert-Higgs mechanism $[13,14]$. In the latter case, there are necessarily several other parameters and degrees of freedom (d.o.f.) associated with $U(1)^{\prime}$ breaking [the dots 


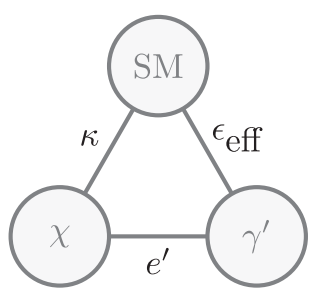

FIG. 1. The three sectors (blobs) and their three connections (lines). In the limit of a massless dark photon, (or more generally, as $\epsilon_{\text {eff }} \rightarrow 0$; see text), the connection between the SM and dark photon blob is absent.

in Eq. (1.1)]. In the sequel, we will assume that these extra ingredients may be neglected. However, we will briefly discuss their possible impact in Appendix B.

In the case of a massless dark photon, the problem of accounting for the DM abundance through the kinetic mixing portal has been studied in Ref. [2]. It has been shown in that work that the observed relic density could be reached along four distinct dynamical mechanisms depending on the values of the parameters of the model. These dynamical mechanisms are freeze-in (regime Ia in the sequel) [2,15-17], reannihilation (IIIa) [2,16], secluded freeze-out (IVa), ${ }^{1}$ and finally, the standard textbook thermal freeze-out mechanism ( $\mathrm{Va}$ and $\mathrm{Vb})$. Altogether, these four mechanisms lead to five different regimes through which the DM abundance can be reached in the case of a massless dark photon. Indeed, the standard freeze-out of the dark matter particles could occur either through annihilation into $\mathrm{SM}$ particles (Vb regime) or into the dark photons themselves (Va regime). We will thus distinguish production mechanisms (classified using roman numerals) and regimes (distinguished using the latin letter $\mathrm{a}$ or $\mathrm{b}$, as in the case of freeze-out).

The aim of the present work is to revisit this classification of DM production mechanisms, taking into account the possible effects of the mass of the dark photon. By considering the dark photon model above, the generic underlying structure we will be considering is that of a system composed of three distinct particle sectors, together with three possible connections between the sectors. This structure is depicted in Fig. 1, again with a focus on the kinetic mixing setup and its ingredients. So, the three sectors (depicted as blobs) consist of the SM, the dark matter particle $\chi$, and the massive dark photon $\gamma^{\prime}$, while the connections between the sectors (depicted as lines) are parametrized by the mixing $\hat{\epsilon}$ (or more precisely $\epsilon_{\text {eff }}$, which will be defined below), the hidden fine structure constant $\alpha^{\prime}$, and the "millicharge" $\kappa$. Considering such a structure, we will point out the existence of four new regimes

\footnotetext{
${ }^{1}$ By secluded freeze-out, we refer here to a scenario in which DM particles freeze out in the dark sector $[18,19]$, but with a temperature $T^{\prime}$ different from the temperature of the SM visible sector, as in Refs. [2,9].
}

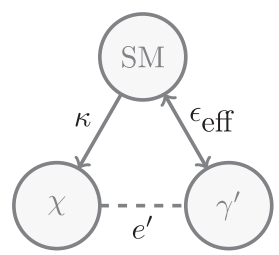

(a) Ia

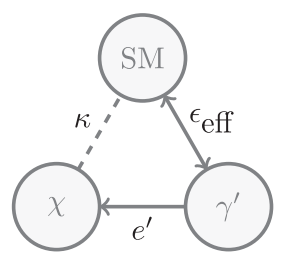

(b) $\mathrm{Ib}$

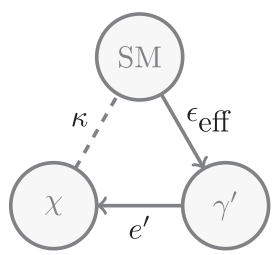

(c) II

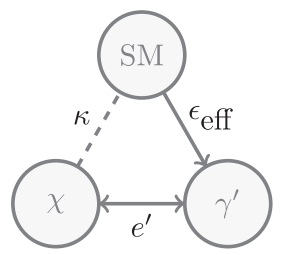

(d) IIIa \& IVa

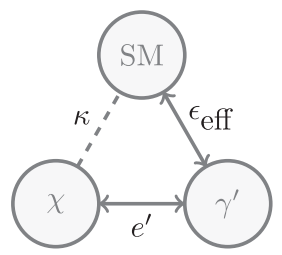

(f) $\mathrm{Va}$

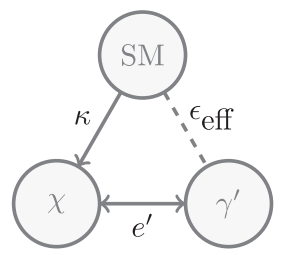

(e) IIIb \& IVb

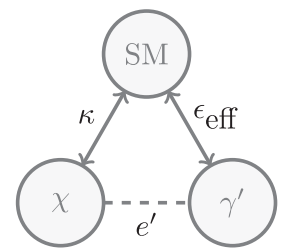

(g) $\mathrm{Vb}$
FIG. 2. The nine possible DM production regimes in the dark photon scenario. A double-sided arrow means that the two corresponding sectors have reached chemical equilibrium; a single-sided arrow indicates slow out-of-equilibrium production of one sector by the other one; a dashed line corresponds to a subdominant interaction between the sectors. Regimes Ia and Ib, II, IIIa and IIIb, IVa and IVb, Va and Vb are associated with five distinct mechanisms to produce the DM abundance: the freeze-in (I), sequential freeze-in (II), reannihilation (III), secluded freezeout (IV) and freeze-out (V) mechanisms, respectively. (see Secs. III A and III B). Notice that the diagrams are identical for the reannihilation and secluded freeze-out mechanisms. Note also that the SM-to- $\gamma^{\prime}$ connection is parametrized by $\epsilon_{\mathrm{eff}}$ when we take into account the thermal corrections. Without such corrections, it is parametrized by $\epsilon$.

(denoted as Ib, II, IIIa, and IVa), including one along a new dynamical mechanism (II), making in total potentially nine distinct ways to reach the observed DM abundance.

As we will explain in the following sections, the nine production regimes can be read from this figure by considering the different ways through which the hiddensector particles can be created and whether they are in thermal equilibrium with each other and/or with the SM sector. In Fig. 2, one sees the four new regimes that emerge in the case of a massive dark photon (and so are absent in 
the massless limit—see later), denoted Ib, II, IIIa, and (a priori; see, however, below) IVa. As the solid arrow lines between the sectors suggest, they all correspond to regimes in which $\mathrm{DM}$ is created from the SM sector via the production of real, on-mass-shell dark photons. All these regimes follow each other along a characteristic pattern in the parameter space, which in the sequel we call "the phase diagram" and that will be displayed later in Sec. III. Most interestingly, some of these new regimes allow for DM production for values of $\kappa$ that are even smaller than in the case of standard freeze-in (regime Ia). As we will see, whether a specific regime is actually relevant will depend not only on the connection parameters, but also on the DM and dark photon masses.

Our work is organized as follows: In Sec. II, we will present how the various particles in the kinetic portal model of Eq. (1.1) can interact, focusing on the case of a massive dark photon. Next, in Sec. III, we will derive and discuss the parameter space or phase diagram in which we show, as a function of the parameters, how the different regimes that lead to the observed DM relic density are related with each other. We discuss each regime in detail but put a particular emphasis on the new regimes. For the sake of our classification and to simplify our discussion, in that section we will be putting aside some complications that arise due to the peculiar nature of the kinetic mixing portal. In particular, we neglect there the impact of thermal effects on the propagation and production of dark photons. These will be considered in Sec. IV, emphasizing their impact on the different production regimes we found. Next, we will discuss briefly in Sec. V the other constraints which hold on this model. Finally, in Sec. VI, we will elaborate on the generality of the phase diagram we have obtained, discussing the possible effect of other d.o.f. and then drawing our conclusions. Appendix A contains a technical summary based on the existing literature on thermal effects on dark photon production and progagation. The case in which the mass of the dark photon arises through the Brout-EnglertHiggs mechanism is considered in Appendix B.

\section{THE THREE SECTORS AND THEIR CONNECTIONS}

We focus on the model of Eq. (1.1) and aim at studying the abundance of the new particles-here, the dark matter, made of particles $\chi$ and $\bar{\chi}$, and the dark photon, $\gamma^{\prime}$. To determine the relevant processes, we need to establish the coupling of dark photons to the SM sector. The procedure is standard and consists first in having canonical kinetic terms for the gauge fields; we repeat the argument here for the sake of clarity. To do so, we exploit the fact that the values of $\epsilon$ that are relevant for DM production will always turn out to be small numbers, so we can treat the effects of mixing as a perturbation. From Eq. (1.1), making the nonorthogonal transformation

$$
B^{\prime \mu} \rightarrow \tilde{B}^{\prime \mu}-\hat{\epsilon} B^{\mu} \quad \text { and } \quad B^{\mu} \rightarrow B^{\mu}
$$

leads to

$$
\begin{aligned}
\mathcal{L} \rightarrow & -\frac{1}{4} \tilde{B}^{\prime \mu \nu} \tilde{B}_{\mu \nu}^{\prime}+\frac{1}{2} m_{\gamma^{\prime}}^{2} \tilde{B}^{\prime \mu} \tilde{B}_{\mu}^{\prime}-\hat{\epsilon} m_{\gamma^{\prime}}^{2} B^{\mu} \tilde{B}_{\mu}^{\prime} \\
& -e^{\prime} \bar{\chi} \gamma^{\mu} \chi\left(\tilde{B}_{\mu}^{\prime}-\hat{\epsilon} B_{\mu}\right)+\cdots
\end{aligned}
$$

to leading order in $\hat{\epsilon}$, where we only show the terms relevant for our argument. The SM particles themselves couple to the SM gauge fields - in particular, the neutral ones, $B^{\mu}=\cos \theta_{W} A_{0}^{\mu}-\sin \theta_{W} Z_{0}^{\mu}$ and $W_{\mu}^{3}=\sin \theta_{W} A_{0}^{\mu}+\cos \theta_{W} Z_{0}^{\mu}$, where $\theta_{W}$ is the SM Weinberg angle; we put the subscripts 0 on $A_{0}^{\mu}$ and $Z_{0}^{\mu}$ to insist on the fact that they are the usual SM fields.

Case $m_{\gamma^{\prime}}=0$ : In the limit $m_{\gamma^{\prime}}=0$, we see from Eq. (2.2) that the SM particles do not couple to the dark photon, identified with $\tilde{B}_{\mu}^{\prime}$, while the $\chi$ particles are coupled to the $\mathrm{SM}$ photon $A_{0}^{\mu}$ with millicharge $\kappa=\epsilon e^{\prime} / e$, where $\epsilon=\hat{\epsilon} \cos \theta_{W}$. They are also coupled vectorially to the $Z_{0}^{\mu}$ boson, with coupling $-e^{\prime} \epsilon \tan \theta_{W}$. Thus, if $m_{\gamma^{\prime}}=0$, the only possibility to create dark photons is to go through the production of $\chi$ particles.

Case $m_{\gamma^{\prime}} \neq 0$ : If instead $m_{\gamma^{\prime}} \neq 0$, dark photons can be created directly by SM particles through the mixing mass term

$$
-\hat{\epsilon} m_{\gamma^{\prime}}^{2} \tilde{B}_{\mu}^{\prime} B^{\mu} \equiv-\epsilon m_{\gamma^{\prime}}^{2} \tilde{B}_{\mu}^{\prime}\left(A_{0}^{\mu}-\tan \theta_{W} Z_{0}^{\mu}\right)
$$

in Eq. (2.2). Like in the description of neutrino oscillations, we refer to the basis of Eq. (2.2) as the interaction eigenstate basis. It is interesting to go forward and diagonalize the mass terms. We will only consider cases in which $m_{B^{\prime}} \ll m_{Z}$, and so again we perform the diagonalization to leading order in $\epsilon \ll 1$. Performing the orthogonal transformation

$$
A^{\prime \mu}=\tilde{B}^{\prime \mu}-\epsilon A_{0}^{\mu} \quad \text { and } \quad A^{\mu}=A_{0}^{\mu}+\epsilon \tilde{B}^{\prime \mu}
$$

transforms Eq. (2.3) into

$$
-\epsilon \tan \theta_{W} m_{\gamma^{\prime}}^{2} A_{\mu}^{\prime} Z_{0}^{\mu}
$$

while $m_{A^{\prime}}=m_{\gamma^{\prime}}$, with $m_{Z_{0}}$ like in the SM. Now, it is easy to see that this mixing term introduces a mass splitting between the $A^{\prime \mu}$ and $Z_{0}^{\mu}$ field that is $\mathcal{O}\left(\epsilon^{2}\right)$ and thus can be neglected. So the dark photon field $A_{\mu}^{\prime}$ has indeed a mass

$$
m_{A^{\prime}}=m_{\gamma^{\prime}},
$$

while the field $A^{\mu}$ is massless. This eigenmass basis makes clear that the particles $\chi$ couple only to the massive dark photon and to the SM $Z$ boson, and not to the massless photon, a fact that is well known in the literature: 


$$
e^{\prime} \bar{\chi} \gamma^{\mu} \chi\left(\tilde{B}_{\mu}^{\prime}-\hat{\epsilon} B_{\mu}\right) \equiv e^{\prime} \bar{\chi} \gamma^{\mu} \chi\left(A_{\mu}^{\prime}+\epsilon \tan \theta_{W} Z_{\mu}\right) .
$$

(We drop the 0 subscript on the $Z$ field from now on as $m_{Z_{0}} \equiv m_{Z}$.) Also, the electrically charged SM particles, like the electron, couple to the dark photon with coupling

$$
e \bar{\psi} \gamma^{\mu} \psi A_{0 \mu} \equiv e \bar{\psi} \gamma^{\mu} \psi\left(A_{\mu}+\epsilon A_{\mu}^{\prime}\right)
$$

The above discussion highlights the fact that, when the dark and SM photons are both massless (and so degenerate), one may define the photon to be the state that couples to SM electrically charged particles $[2,20]$, whereas in the massive case the photon does not couple to the DM. It however raises the question of how to take the limit $m_{\gamma^{\prime}} \rightarrow 0$. This will be addressed in Sec. IV. In the meantime, we consider the case $m_{\gamma^{\prime}} \neq 0$.

In the model just introduced, we must thus consider the existence of three distinct populations (or sectors, or reservoirs) of particles in the early stages of the Universe. The main reservoir consists of the SM particles; they will be assumed to be in thermal equilibrium at a temperature $T$. The hidden sector consists on one side of the $\chi$ particles and their antiparticles (DM) and, on the other side, of the dark photons. These two populations do not have to be necessarily in thermal equilibrium with each other or with the SM sector. Their respective abundance depends then on how the three reservoirs are connected with each other. The dominant processes directly connecting the SM and DM populations are the pair annihilation of SM particles (or Z-boson decay for some mass range) into DM pairs [2]. The strength of these processes is essentially set by a single parameter (the "millicharge"),

$$
\kappa \equiv \epsilon \sqrt{\alpha^{\prime} / \alpha}
$$

The processes connecting the SM reservoir and the dark photon population are, on the other hand, determined by the $\epsilon$ parameter; these a priori include bremsstrahlung $e^{ \pm} e^{ \pm} \rightarrow$ $e^{ \pm} e^{ \pm} \gamma^{\prime}$ (which may be neglected), Compton scattering $e^{ \pm} \gamma \rightarrow e^{ \pm} \gamma^{\prime}$, pair annihilation $e^{+} e^{-} \rightarrow \gamma \gamma^{\prime}$, and coalescence $e^{+} e^{-} \rightarrow \gamma^{\prime}$. They involve distinct powers of $\alpha$ but also distinct temperature dependence and so are dominant in different mass and temperature regimes. As for the processes connecting the DM and dark photon populations, they are set by $\alpha^{\prime}$ and are dominated (at lowest order in $\alpha^{\prime}$ ) by $\gamma^{\prime} \gamma^{\prime} \leftrightarrow \chi \bar{\chi}$. The Feynman diagrams for all these connecting processes are shown in Fig. 3. This structure leads to the diagram of Fig. 1, where the blobs represent schematically the reservoirs, and the lines the possible connections. In the massless case, the triangle of Fig. 1 has one connecting edge fewer, since there is no direct connection between the hidden photon and SM populations. This, in brief, explains why there are more DM production regimes in the massive case than in the massless case.

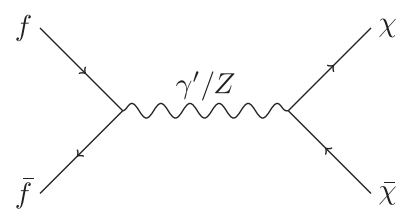

(a)

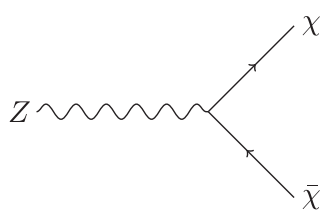

(b)

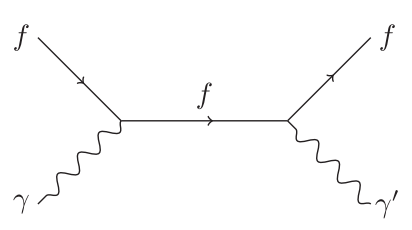

(c)

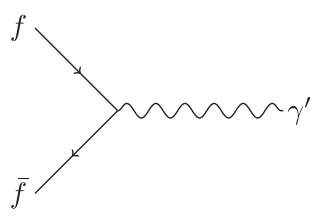

(e)

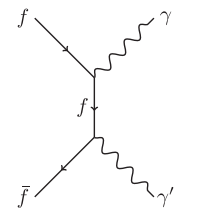

(d)

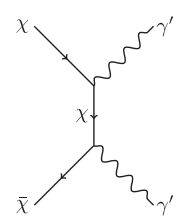

(f)
FIG. 3. Feynman diagrams for all the connecting processes between the three populations.

\section{PHASE DIAGRAM OF DARK MATTER PRODUCTION}

We now turn to the systematic discussion of the dependence of the DM relic abundance on the connector parameters, starting from an empty reservoir in the dark sector, passing through all the regimes, up to a dark sector that is fully in thermal equilibrium with the SM. ${ }^{2}$

For the sake of clarity and generality, we will not include in this section any of the thermal effects that are specific to the dark photon setup. Instead, thermal effects on dark photon production, which can be very important in some cases as we shall see, will be determined and discussed in Sec. IV. Also, in this section and the sequel, we will rely heavily on the results obtained in Ref. [2], to which we refer for more in-depth discussions of some of the production regimes, including explicit expressions for the relevant cross sections (given in that reference for a massless dark photon).

The DM abundance depends on the DM and dark photon masses, and on two among the three connector parameters $\kappa, \alpha^{\prime}$, and $\epsilon_{\text {eff }}$, since only two are independent; see Eq. (2.9). Thus, for a given set of $\chi$ and $\gamma^{\prime}$ masses, one can, for instance, give the relic abundance as contour lines in the $\kappa-\alpha$ plane. This representation leads to a "phase diagram" that displays the various regimes. Note importantly that for

\footnotetext{
${ }^{2}$ If the visible and hidden sectors were feebly coupled with each other, it would not be surprising that the reheating at the end of inflation took place mostly in one of the sectors rather than both of them. We assume here that the reheating happened in the visible sector.
} 


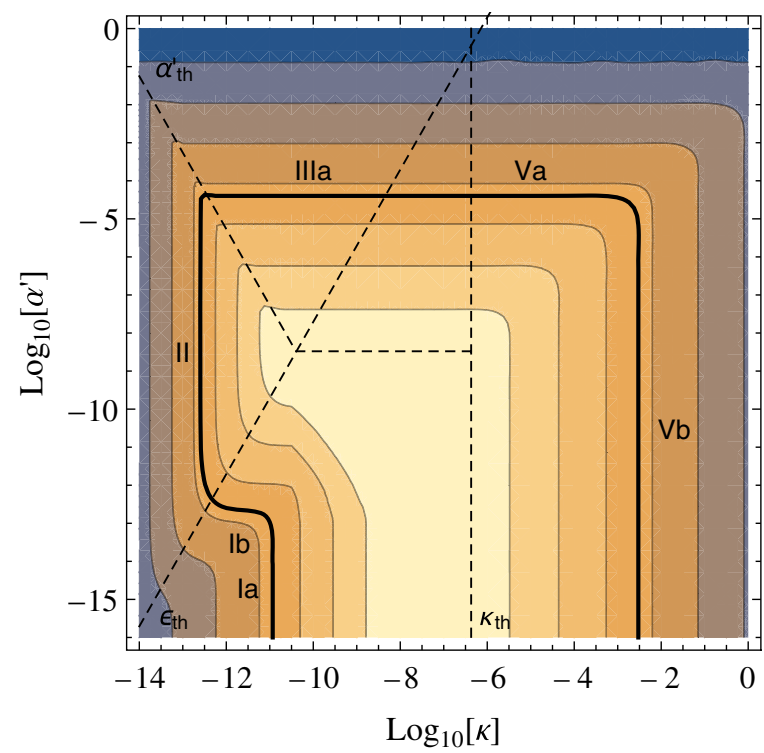

FIG. 4. DM relic density obtained as a function of $\kappa$ and $\alpha^{\prime}$ for $m_{\mathrm{DM}}=3 \mathrm{GeV}$ and $m_{\gamma^{\prime}}=1 \mathrm{GeV}$. This diagram displays the various different production regimes which can lead to the observed relic density without taking thermal effects into account. For this particular choice of masses, one obtains six different production regimes along four dynamical mechanisms: freeze-in (Ia and Ib), sequential freeze-in (II), reannihilation (IIIa), and freeze-out (Va and $\mathrm{Vb}$ ).

all the discussion below we will always assume that $m_{\gamma^{\prime}}<m_{\mathrm{DM}}$, so that DM is always kinematically free to annihilate into dark photons. The opposite case $m_{\gamma^{\prime}}>m_{\mathrm{DM}}$ leads to a different (and simpler) phenomenology that we will not discuss here. The phase diagram comes out from integrating a set of Boltzmann equations that determine the evolution of the DM and $\gamma^{\prime}$ yields as a function of the visible sector temperature. For the case of a massless dark photon, the contour lines of constant DM relic density in the phase diagram have roughly the shape of a rectangle (dubbed the "mesa" in Ref. [2]), displaying five regimes, along four dynamical production mechanisms. These four mechanisms are freeze-in (Ia in the classification of Fig. 2), reannihilation (IIIb), secluded freeze-out (IVb), and ordinary freeze-out (with freeze-out either to dark photons, Va, or to $\mathrm{SM}$ particles, $\mathrm{Vb}$ ), leading altogether to five distinct regimes. Starting with an empty hidden sector, the DM abundance is reached through the following sequence of regimes:

$$
\mathrm{Ia} \rightarrow \mathrm{IIIb} \rightarrow \mathrm{IVb} \rightarrow \mathrm{Va} \rightarrow \mathrm{Vb} \quad\left(m_{\gamma^{\prime}}=0\right) .
$$

In particular, the freeze-in regime, Ia, proceeds through slow $\kappa$-driven $\mathrm{SM} \rightarrow \mathrm{DM}$ processes and leads to a vertical line at small $\kappa$ in the "mesa" structure of the phase diagram; see Ref. [2]. When the dark photon mass matters, this simple vertical line structure does not hold anymore, and

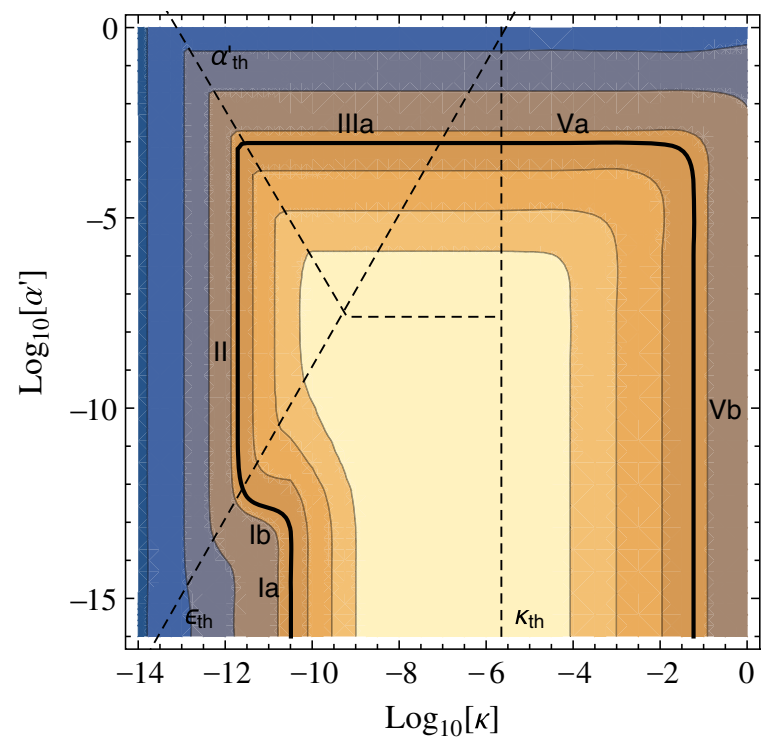

FIG. 5. Same as Fig. 4, but with $m_{\mathrm{DM}}=100 \mathrm{GeV}$ and $m_{\gamma^{\prime}}=10 \mathrm{GeV}$.

instead, one has a more complicated structure for small $\kappa$. Actually, one can distinguish four more regimes for values of $\kappa$ that are small enough so that the $\mathrm{SM} \rightarrow$ DM processes do not thermalize. This is illustrated by the phase diagram depicted in Fig. 4, in which we get for one example set of masses $m_{\mathrm{DM}}=3 \mathrm{GeV}$ and $m_{\gamma^{\prime}}=1 \mathrm{GeV}$. Much heavier DM candidates are of course possible, as shown in Fig. 5, for $m_{\mathrm{DM}}=100 \mathrm{GeV}$ and $m_{\gamma^{\prime}}=10 \mathrm{GeV}$. More to the point, the phase diagram, which shows contour lines of constant DM relic density, has a distinct shape: the "mesa" has an extension toward much smaller values of the $\kappa$ parameter, suggesting the shape of a "mooring bollard." These features, and the corresponding new regimes, are, as we shall see, due to the possibility of dark photon production of DM.

The most generic sequence of regimes appearing along the mooring bollard pattern is the one appearing in Figs. 4 and 5 - that is to say,

$$
\mathrm{Ia} \rightarrow \mathrm{Ib} \rightarrow \mathrm{II} \rightarrow \mathrm{IIIa} \rightarrow \mathrm{Va} \rightarrow \mathrm{Vb} \quad\left(m_{\gamma^{\prime}} \neq 0\right) .
$$

This structure, on top of the regimes already existing in the massless case (Ia, Va, and $\mathrm{Vb}$ ), involve three new regimes: Ib, II, and IIIa. The fourth new regime, IVa, as well as the two other regimes already existing in the massless case (IIIb and IVb), can appear for other choices of the parameter and more generally for other models. The four new regimes arise from the extra SM production of dark photons. In all cases, the mooring bollard shape of the phase diagram is a generic signature.

The general set of Boltzmann equations that determine the evolution of the DM and $\gamma^{\prime}$ abundances $\left(Y_{\mathrm{DM}, \gamma^{\prime}}=n_{\mathrm{DM}, \gamma^{\prime}} / s\right.$, with $s$ being the entropy density and 
$\left.n_{\mathrm{DM}}=n_{\chi}+n_{\bar{\chi}}\right)$ as a function of time, and therefore lead to the phase diagram, takes the form ${ }^{3}$

$$
\begin{aligned}
z H s \frac{d Y_{\mathrm{DM}}}{d z}= & \left\langle\sigma_{\mathrm{DM} \rightarrow \mathrm{SM}} v\right\rangle\left[\left(n_{\mathrm{DM}}^{\mathrm{eq}}\right)^{2}-n_{\mathrm{DM}}^{2}\right] \\
& +\left\langle\Gamma_{\mathrm{DM} \rightarrow \mathrm{SM}}^{D}\right\rangle \frac{n_{\mathrm{Z}}^{\mathrm{eq}}}{\left(n_{\mathrm{DM}}^{\mathrm{eq}}\right)^{2}}\left[\left(n_{\mathrm{DM}}^{\mathrm{eq}}\right)^{2}-n_{\mathrm{DM}}^{2}\right] \\
& +\left\langle\sigma_{\gamma^{\prime} \rightarrow \mathrm{DM}} v\right\rangle n_{\gamma^{\prime}}^{2}-\left\langle\sigma_{\mathrm{DM} \rightarrow \gamma^{\prime}} v\right\rangle n_{\mathrm{DM}}^{2}, \\
z H s \frac{d Y_{\gamma^{\prime}}}{d z}= & \left\langle\sigma_{\gamma^{\prime} \rightarrow \mathrm{SM}} v\right\rangle n_{\mathrm{SM}}^{\mathrm{eq}}\left[n_{\gamma^{\prime}}^{\mathrm{eq}}-n_{\gamma^{\prime}}\right] \\
& +\left\langle\sigma_{\mathrm{DM} \rightarrow \gamma^{\prime}} v\right\rangle n_{\mathrm{DM}}^{2}-\left\langle\sigma_{\gamma^{\prime} \rightarrow \mathrm{DM}} v\right\rangle n_{\gamma^{\prime}}^{2},
\end{aligned}
$$

where $H$ is the Hubble parameter and $z=m_{\mathrm{DM}} / T$, with $T$ being the temperature of the visible sector. The quantity $\Gamma^{D}$ refers to the $Z$ decay rate into a pair of DM particles. In writing these Boltzmann equations, we assume that the dark photon is lighter than the dark matter. ${ }^{4}$ A sum over the different $\mathrm{SM} \leftrightarrow \mathrm{DM}$ and $\mathrm{SM} \leftrightarrow \gamma^{\prime}$ channels is implicit everywhere in these equations. In the sequel, so as to avoid cluttering of the equations, we will regroup the scattering and decay terms involving SM particles into

$$
\gamma_{\mathrm{SM} \leftrightarrow \mathrm{DM}}^{\mathrm{eq}}=\left\langle\sigma_{\mathrm{DM} \rightarrow \mathrm{SM}} v\right\rangle\left(n_{\mathrm{DM}}^{\mathrm{eq}}\right)^{2}+\left\langle\Gamma_{\mathrm{SM} \rightarrow \mathrm{DM}}^{D}\right\rangle n_{\mathrm{SM}}^{\mathrm{eq}}
$$

and

$$
\gamma_{\mathrm{SM} \leftrightarrow \gamma^{\prime}}^{\mathrm{eq}}=\left\langle\sigma_{\gamma^{\prime} \rightarrow \mathrm{SM}} v\right\rangle n_{\mathrm{SM}}^{\mathrm{eq}} n_{\gamma^{\prime}}^{\mathrm{eq}} .
$$

Although the Boltzmann equations contain many terms, for most production regimes, only one or two of these terms are relevant. As we will also see, these equations are not sufficient to correctly determine the amount of DM produced in the reannihilation regimes, which are characterized by hidden and visible sectors with distinct temperatures: in these cases, one also needs to evaluate the energy that has been transferred from the SM to the hidden-sector particles.

To understand the distinction between the various production regimes, it is useful to start by delimiting the regions of parameter space depending on whether or not the various connecting processes lead to thermalization. To determine whether the DM particles thermalize with the SM thermal bath, we use the simple criteron

$$
\left.\frac{\Gamma_{\mathrm{SM} \leftrightarrow \mathrm{DM}}}{H}\right|_{T \sim m_{\mathrm{DM}}} \gtrsim 1,
$$

\footnotetext{
${ }^{3}$ Here and in subsequent Boltzmann equations, we have included factors of $1 / 2$, typical of Dirac DM particles, in the definitions of the cross sections [21].

${ }^{4}$ Thus, we do not consider the scenario in which the dark photons produced through freeze-in subsequently decay into pairs of DM particles [22-24].
}

with $\Gamma_{\mathrm{SM} \leftrightarrow \mathrm{DM}}=\gamma_{\mathrm{SM} \leftrightarrow \mathrm{DM}}^{\mathrm{eq}} / n_{\mathrm{DM}}^{\mathrm{eq}}(z)$. This leads to the following condition on the millicharge parameter:

$$
\kappa \gtrsim \kappa_{\mathrm{th}} \equiv 3.8 \times 10^{-7}\left(\frac{m_{\mathrm{DM}}}{\mathrm{GeV}}\right)^{1 / 2},
$$

which is depicted by a vertical line in Figs. 4 and 5. As for the thermalization of the dark photons with the SM thermal bath, what matters typically is if thermalization (which leads to $E_{\gamma^{\prime}} \sim T$ ) has occurred by the time the DM number freezes, $T \sim m_{\mathrm{DM}}$. Thus, similarly to the condition above, we use $\mathrm{u}^{5}$

$$
\left.\frac{\Gamma_{\mathrm{SM} \leftrightarrow \gamma^{\prime}}}{H}\right|_{T \simeq m_{\mathrm{DM}}} \gtrsim 1
$$

as the condition for the thermalization of the dark photons with the SM, which numerically translates into a condition on the (in principle effective, but we remind the reader that we neglect the thermal effects for the time being) mixing parameter,

$$
\epsilon \gtrsim \epsilon_{\text {th }} \equiv 4.1 \times 10^{-8}\left(\frac{m_{\mathrm{DM}}}{\mathrm{GeV}}\right)^{1 / 2},
$$

with $\Gamma_{\mathrm{SM} \leftrightarrow \gamma^{\prime}}=\gamma_{\mathrm{SM} \leftrightarrow \gamma^{\prime}}^{\mathrm{eq}} / n_{\gamma^{\prime}}^{\mathrm{eq}}(z)$. As $\kappa=\epsilon \sqrt{\alpha^{\prime} / \alpha}$, this condition corresponds to a diagonal dashed line in the phase diagram; see Figs. 4 and 5. As for the thermalization between the dark photons and DM particles themselves, it depends on the scenario considered. We now discuss each of the new regimes, using the phase diagram of Fig. 4 as reference.

\section{A. Freeze-in: Regimes Ia and Ib}

The Ia freeze-in regime corresponds to the production of DM particles directly from the SM particles, through outof-equilibrium processes $\mathrm{SM} \rightarrow \mathrm{DM}$ parametrized by $\kappa$. In this regime, $\alpha^{\prime}$ is assumed to be too small for the processes $\gamma^{\prime} \rightarrow$ DM to play any role. Thus, in Eq. (3.3), only the $\mathrm{SM} \rightarrow \mathrm{DM}$ term is relevant for producing the DM.

$$
\underline{\text { Regime Ia }}: \quad z H s \frac{d Y_{\mathrm{DM}}}{d z} \approx \gamma_{\mathrm{SM} \leftrightarrow \mathrm{DM}}^{\mathrm{eq}}(z) .
$$

For fixed DM mass, the production depends only on the parameter $\kappa$, so the observed relic abundance is given by a

\footnotetext{
${ }^{5}$ Notice that relativistic species do not reach kinetic equilibrium long before they enter into chemical equilibrium [2]. In particular, the kinetic $\chi S M \rightarrow \chi S M$ and chemical $S M S M \rightarrow \chi \chi$ processes enter into equilibrium at about the same time, because they involve relativistic particles. As for the elastic scattering processes $\gamma^{\prime} f \rightarrow \gamma^{\prime} f$, which could bring $\gamma^{\prime}$ into kinetic equilibrium, they are suppressed by an extra power of $\epsilon^{2}$ with respect to $\gamma^{\prime} f \rightarrow \gamma f$.
} 
vertical line in the phase diagram, corresponding to the lower-left corner in Fig. 4.

Note that for the small value of $\alpha^{\prime}$, and with the value of $\kappa$ that this freeze-in regime requires, $\kappa \sim 10^{-10}$, values of $\epsilon$ lie above the critical value $\epsilon_{\text {th }}$ for the thermalization of dark photons [Eq. (3.10)]. Thus, unlike for the SM $\rightarrow$ DM freeze-in scenario in the massless dark photon case, in this instance the dark photons thermalize with the SM sector, forming a single thermal bath characterized by the temperature $T$. However, this thermalization does not change anything in the dynamics of this freeze-in regime as a function of $T$, since the DM particles are created dominantly by the SM particles. The only effect is that the thermalized $\gamma^{\prime}$ (dark photons) modify the number of relativistic d.o.f. and thus the Hubble expansion rate. The modification is of order $g_{\gamma}^{\prime} / g_{\star}^{\mathrm{SM}}=2 / g_{\star}^{\mathrm{SM}} \sim 10^{-2}$, a small effect that we neglect in Fig. 4.

In Fig. 6, we show as a function of $m_{\mathrm{DM}}$ the $\kappa$ required to reach the observed relic abundance in the Ia regime, which we denote as $\kappa_{\text {Ia }}$. Effectively, as the thermalized dark photons play a negligible role in the production of the DM particles, this curve is essentially (modulo a slight dependence on the dark photon mass in the dark photon propagator), the same as in the massless dark photon case [2]. The dependence of $\kappa_{\text {Ia }}$ on $m_{\mathrm{DM}}$ is complicated by the fact that the dominant production channels depend also on $m_{\mathrm{DM}}$, but otherwise the number of DM particles created through $\mathrm{SM}_{\mathrm{i}} \mathrm{SM}_{\mathrm{i}} \rightarrow \mathrm{DMDM}$ is simply related to equilibrium quantities evaluated at a temperature $T \ll \max \left\{m_{i}, m_{\mathrm{DM}}\right\}$,

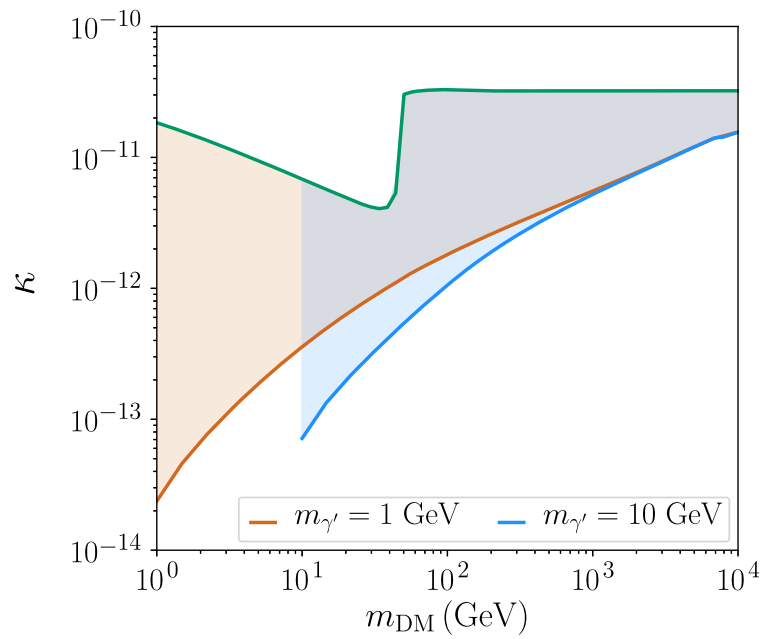

FIG. 6. Values of $\kappa$ needed to account for the observed relic density, as a function of $m_{\mathrm{DM}}$, for the standard freeze-in (regime Ia, green line) and for the sequential freeze-in (regime II, orange line for $m_{\gamma^{\prime}}=1 \mathrm{GeV}$ and blue line for $m_{\gamma^{\prime}}=10 \mathrm{GeV}$ ) in the $\kappa-m_{\mathrm{DM}}$ plane. The shaded regions thus correspond to regime $\mathrm{Ib}$ - that is, freeze-in from thermalized dark photons. We consider $m_{\gamma^{\prime}}<m_{\mathrm{DM}}$.
$Y_{\mathrm{DM}}(z)=\left.\sum_{i} c_{i} \frac{\left(n_{i}^{\mathrm{eq}}\right)^{2}\left\langle\sigma_{\mathrm{SM}_{\mathrm{i}} \rightarrow \mathrm{DM}} v\right\rangle}{H s}\right|_{T=\max \left\{T, m_{i}, m_{\mathrm{DM}}\right\}}$,

where the $c_{i}$ 's are coefficients of order unity that depend on the channel considered [2].

Going up along the vertical line of the Ia regime depicted in Fig. 4, the value of $\alpha^{\prime}$ increases while that of $\epsilon$ decreases. Thus, at some point, $\epsilon$ becomes smaller than $\epsilon_{\text {th }}$ (given in Fig. 4 by the diagonal dashed line). However, before this could happen and while the dark photons are still in thermal equilibrium with the SM, $\alpha^{\prime}$ becomes large enough for the thermal dark photons to sizeably pair-produce DM particles. So, in this case, DM becomes dominantly produced through $\gamma^{\prime} \rightarrow \mathrm{DM}$ freeze-in instead of SM $\rightarrow$ DM freeze-in. Clearly, in this case, a smaller value of $\kappa$ is required to avoid the overproduction of DM particles and as a result, the abundance depends only on $\alpha^{\prime}$, giving rise to the horizontal line depicted in Fig. 4. This "freeze-in from dark photons" regime is denoted as $\mathrm{Ib}$ in this figure. ${ }^{6}$ For this regime, the only relevant term in the Boltzmann equation is the one producing DM from dark photons:

$$
\underline{\text { Regime Ib }: ~} \quad z H s \frac{d Y_{\mathrm{DM}}}{d z} \approx \gamma_{\gamma^{\prime} \leftrightarrow \mathrm{DM}}^{\mathrm{eq}},
$$

with $\gamma_{\gamma^{\prime} \leftrightarrow \mathrm{DM}}^{\mathrm{eq}}=\left\langle\sigma_{\gamma^{\prime} \leftrightarrow \mathrm{DM}} v\right\rangle\left(n_{\gamma^{\prime}}^{\mathrm{eq}}\right)^{2}$, so that the DM yield is simply given by

$$
Y_{\mathrm{DM}}(z)=\left.c_{\gamma^{\prime}} \frac{\left(n_{\gamma^{\prime}}^{\mathrm{eq}}\right)^{2}(z)\left\langle\sigma_{\gamma^{\prime} \rightarrow \mathrm{DM}} v\right\rangle}{H s}\right|_{T=\mathrm{Max}\left[T, m_{\mathrm{DM}}\right]},
$$

with $c_{\gamma^{\prime}}=\mathcal{O}(1)$. In the limit $m_{\gamma^{\prime}} \ll m_{\mathrm{DM}}$, we found that the value of $\alpha^{\prime}$ required to create the right amount of DM particles out of equilibrium from the $\gamma^{\prime}$ is

$$
\alpha_{\mathrm{Ib}}^{\prime}=2.5 \times 10^{-13}\left(\sqrt{g_{*}\left(m_{\mathrm{DM}}\right)} g_{*, S}\left(m_{\mathrm{DM}}\right)\right)^{1 / 2},
$$

where the dependence on the in-equilibrium SM d.o.f. stems from the $H s$ factor in the denominator of Eq. (3.14). As stated above, the value of $\kappa$ in the Ib regime must be below that of Ia, $\kappa_{\text {Ia }}$, so as to avoid the overproduction of DM. However, if it becomes much smaller than $\kappa_{\text {Ia }}$, we enter in yet another regime, which we now discuss.

\section{B. Sequential freeze-in: Regime II}

As we move along the $\mathrm{Ib}$ regime line toward smaller values of $\kappa$ in Fig. 4, the value of $\epsilon$ decreases. Thus, at some

\footnotetext{
${ }^{6}$ This regime has been briefly discussed in Ref. [25], where it is dubbed "inverse annihilation," and in Ref. [26], in a model with a scalar singlet that mixes with the Higgs. It is also considered in a scenario with a $Z^{\prime}$ based on a $B-L$ gauge symmetry, which appeared simultaneously with our work [27].
} 
point $\epsilon$ becomes smaller than $\epsilon_{\text {th }}$; i.e., the dark photons no longer thermalize with the SM. At this point, clearly Eq. (3.13) does not apply, since $n_{\gamma^{\prime}}$ is no longer equal to its equilibrium value $n_{\gamma^{\prime}}^{\text {eq }}$. Thus, the system enters a new regime in which none of the processes induced by $\kappa, \epsilon$, or $\alpha^{\prime}$ have ever been in thermal equilibrium. Also, the SM $\rightarrow$ DM freeze-in processes induced by $\kappa$ are clearly too slow to be efficient, since $\kappa \ll \kappa_{\text {Ia }}$. However, it turns out that the slow out-of-equilibrium production of dark photons from the SM (which is controlled by $\epsilon$ ) followed by the slow outof-equilibrium production of DM by these unthermalized dark photons can produce enough DM. We thus have a chain of successive freeze-in processes, something we dub "sequential freeze-in"; see regime II in Fig. 4. As DM $\rightarrow \gamma^{\prime}$ and $\gamma^{\prime} \rightarrow$ SM processes have a negligible impact in this regime, they drop from the Boltzmann equations, which take the form

$$
\begin{gathered}
\frac{\text { Regime II }}{z H s} z H \frac{d Y_{\gamma^{\prime}}}{d z} \approx \gamma_{\mathrm{SM} \leftrightarrow \gamma^{\prime}}^{\mathrm{eq}}, \\
z H s \frac{d Y_{\mathrm{DM}}}{d z} \approx \gamma_{\gamma^{\prime} \leftrightarrow \mathrm{DM}},
\end{gathered}
$$

where $\gamma_{\gamma^{\prime} \leftrightarrow \mathrm{DM}}=\left\langle\sigma_{\gamma^{\prime} \leftrightarrow \mathrm{DM}} v\right\rangle\left(n_{\gamma^{\prime}}\right)^{2}$. With respect to the equilibrium reaction density, this reaction density is suppressed by a $\left(n_{\gamma^{\prime}} / n_{\gamma^{\prime}}^{\mathrm{eq}}(z)\right)^{2}$ factor, with $n_{\gamma^{\prime}}$ determined by the first Boltzmann equation [Eq. (3.16)]. As the number of dark photons produced by SM particles is proportional to $\epsilon^{2}$, the number of DM particles, which is proportional to $n_{\gamma^{\prime}}^{2}\left\langle\sigma_{\gamma^{\prime} \rightarrow \mathrm{DM}} v\right\rangle$, is $\propto \epsilon^{4} \alpha^{\prime 2} \sim \kappa^{4}$. Thus, in the sequential freeze-in regime, the relic density depends only on $\kappa$ (as in Ia freeze-in regime, albeit with a smaller value of $\kappa<\kappa_{\text {Ia }}$ ) and corresponds to a vertical line in the phase diagram, as seen in Fig. 4.

The line separating regimes Ib and II is the line for which $\epsilon=\epsilon_{\text {th }}$. Consequently, the value of $\kappa$ required to reach the DM abundance in regime II is set by setting $\epsilon=\epsilon_{\text {th }}$ while taking $\alpha^{\prime}=\alpha_{\mathrm{Ib}}^{\prime}$ for the regime Ib [Eq. (3.15)]. For example, for the masses considered in Fig. 4, this gives

$$
\kappa_{\mathrm{II}}=\epsilon_{\mathrm{th}} \sqrt{\frac{\alpha_{\mathrm{Ib}}^{\prime}}{\alpha}} \simeq 10^{-13} \ll \kappa_{\mathrm{Ia}},
$$

in good agreement with the numerical value shown in this figure. This shows that along sequential freeze-in it is possible to have a SM-to-DM connection (i.e., millicharge $\kappa)$ which may be orders of magnitude weaker than along the ordinary freeze-in regime: for the example of Fig. $4, \kappa_{\text {II }}$ is 3 orders of magnitude smaller than $\kappa_{\text {Ia }}$.

We give in Fig. 6 the value $\kappa_{\mathrm{II}}$ as a function of $m_{\mathrm{DM}}$ corresponding to the observed DM relic density. This is aimed at illustrating the fact that sequential freeze-in requires a smaller value of $\kappa$. Thermal effects below will somehow change the details of the picture, but not this overall conclusion, which we deem to be general for the model with relatively light mediators. Figure 6 also shows that, for large values of $m_{\mathrm{DM}} / m_{\gamma^{\prime}}, \kappa_{\mathrm{II}}$ tends to $\kappa_{\mathrm{Ia}}$, meaning that the only relevant mechanism to produce DM in this case is regime Ia in this region of parameter space. This merging of regime II (and thus of the intermediate regime $\mathrm{Ib}$ too) with the ordinary Ia freeze-in regime for large values of $m_{\mathrm{DM}} / m_{\gamma^{\prime}}$ is due to the fact that more massive DM candidates are produced at higher temperatures, and thus at earlier times. If the dark photons have less time to thermalize with the SM, they will need a comparatively larger mixing to achieve it. Thus, the turning from phase Ib to II occurs for a larger value of $\kappa$, making $\kappa_{\text {II }}$ get closer to $\kappa_{\text {Ia }}{ }^{7}$

\section{Reannihilation: Regimes IIIa and IIIb}

Moving in Fig. 4 along regime II toward larger values of $\alpha^{\prime}$, clearly, at some point $\alpha^{\prime}$ becomes large enough for the DM and the dark photon to reach thermal equilibrium so that the system enters a new regime called reannihilation. As explained at length in Ref. [2], this regime holds if two conditions are fulfilled. First, it requires that thermalization of the dark sector occurs without thermalization with the SM sector. Consequently, the system is composed of two thermal baths (the SM and the hidden sector) characterized by two different temperatures, $T$ and $T^{\prime}$ with $T>T^{\prime}$. Second, this regime requires that the slow outof-equilibrium production of dark sector particles from the SM ones still be efficient at the time of freeze-out of the DM into hidden-sector particles (which takes place at a temperature $T^{\prime} \leq m_{\mathrm{DM}}$ ). In this case, DM freezes later than in the case of a standard freeze-out in the hidden sector (i.e., a "secluded freeze-out"; see below) and undergoes a period of reannihilation.

In the present case, reannihilation could be achieved in two distinct ways, depending on whether the hidden sector is populated through SM $\rightarrow \gamma^{\prime}$ (controlled by $\epsilon$ ) or through $\mathrm{SM} \rightarrow \mathrm{DM}$ (controlled by $\kappa$ ) slow processes. These regimes are denoted as IIIIa and IIIb, respectively, in Figs. 2(d), 2(e), and 4. Now, in practice, if one moves toward larger values of $\alpha^{\prime}$ along the sequential freeze-in regime (regime II) line in Fig. 4, the system enters into regime IIIa and not regime IIIb. This simply stems from the fact that, with a value of $\kappa$ as small as the one which holds for regime II and which is smaller than for the ordinary $\mathrm{SM} \rightarrow \mathrm{DM}$ freeze-in Ia regime, DM particles cannot be sizeably produced through $\mathrm{SM} \rightarrow \mathrm{DM}$ processes. Consequently, we begin with regime IIIa and discuss next regime IIIb.

The value of $\alpha^{\prime}$ for which the transition from II to IIIa regimes occurs corresponds to the minimum value of $\alpha^{\prime}$ for

\footnotetext{
${ }^{7}$ When we take into account the finite-temperature corrections, we will see that the merging of regimes $\kappa_{\text {II }}$ and $\kappa_{\text {Ia }}$ occurs for somewhat smaller values of $m_{\mathrm{DM}} / m_{\gamma^{\prime}}$; see Sec. IV.
} 
which the $\mathrm{DM} \leftrightarrow \gamma^{\prime}$ processes thermalize before the number of DM particles freezes. This is determined by requesting that the rate for $\gamma^{\prime} \rightarrow \mathrm{DM}$ be larger than the Hubble rate when the out-of-equilibrium SM $\rightarrow \gamma^{\prime}$ source stops creating dark photons with an energy large enough for the $\gamma^{\prime} \rightarrow$ DM process to proceed-that is, when $T \sim m_{\mathrm{DM}}$,

$$
\left.\frac{\left\langle\sigma_{\gamma^{\prime} \rightarrow \mathrm{DM}} v\right\rangle n_{\gamma^{\prime}}}{H}\right|_{T \simeq m_{\mathrm{DM}}} \gtrsim 1 .
$$

In this equation, the number density of dark photons that have been created out of equilibirium from the SM, $n_{\gamma^{\prime}}$, can be related to their number density if they were in thermal equilibrium with the SM thermal bath, $n_{\gamma^{\prime}}^{\text {eq }}$, multiplying it by the square of the ratio between $\epsilon$ and the value $\epsilon_{\mathrm{th}}$ that leads to thermalization, given in Eq. (3.10), so that $n_{\gamma^{\prime}}=\left(\epsilon / \epsilon_{\mathrm{th}}\right)^{2} n_{\gamma^{\prime}}^{\mathrm{eq}}(T)$. Thus, the value of $\alpha_{\text {IIIa }}^{\prime}$ at the II-toIIIa transition follows from the one required for the DM $\leftrightarrow$ $\gamma^{\prime}$ processes to thermalize if the dark photon was in thermal equilibrium with the SM, as

$$
\alpha_{\text {IIIa }}^{\prime}=\frac{\epsilon_{\mathrm{th}}}{\epsilon} \alpha_{\mathrm{th}}^{\prime} .
$$

This gives

$$
\alpha_{\mathrm{th}}^{\prime} \approx 2 \times 10^{-9}\left(\frac{m_{\mathrm{DM}}}{\mathrm{GeV}}\right)^{1 / 2} .
$$

Once the dark photons and the DM particles have thermalized, the abundance of $\gamma^{\prime}$ and DM particles are governed by the following set of Boltzmann equations:

$$
\begin{aligned}
& \underline{\text { Regime IIIa }}: z H s \frac{d Y_{\gamma^{\prime}}}{d z}=\gamma_{\mathrm{SM} \leftrightarrow \gamma^{\prime}}^{\mathrm{eq}}(z) \\
& -\gamma_{\gamma^{\prime} \leftrightarrow \mathrm{DM}}^{\mathrm{eq}}\left(z^{\prime}\right)\left[1-\left(\frac{Y_{\mathrm{DM}}}{Y_{\mathrm{DM}}^{\mathrm{eq}}\left(z^{\prime}\right)}\right)^{2}\right], \\
& z H s \frac{d Y_{\mathrm{DM}}}{d z}=\gamma_{\gamma^{\prime} \leftrightarrow \mathrm{DM}}^{\mathrm{eq}}\left(z^{\prime}\right)\left[1-\left(\frac{Y_{\mathrm{DM}}}{Y_{\mathrm{DM}}^{\mathrm{eq}}\left(z^{\prime}\right)}\right)^{2}\right] .
\end{aligned}
$$

The $\gamma^{\prime} \leftrightarrow$ DM terms are responsible for the thermalization between the $\gamma^{\prime}$ and DM particles. They account for the fact that the $\gamma^{\prime}$ number density is the one at equilibrium taken at temperature $T^{\prime}$, i.e., $Y_{\gamma^{\prime}}^{\mathrm{eq}}\left(z^{\prime}\right)$, so that the corresponding reaction rate is $\gamma_{\gamma^{\prime} \leftrightarrow \mathrm{DM}}^{\mathrm{eq}}\left(z^{\prime}\right)$. The first term of the first equation describes the slow production of dark photons from the SM.

The above Boltzmann equations can be solved provided we know $T^{\prime}$ as a function of $T$. This is determined from integrating the Boltzmann equation for the SM-to-hiddensector energy $\operatorname{transfer}^{8}[2]$ :

\footnotetext{
${ }^{8} \mathrm{We}$ neglect in this equation the subdominant elastic process $\gamma^{\prime} f \rightarrow \gamma^{\prime} f$; see footnote 5 .
}

$$
\begin{aligned}
z H \frac{d \rho^{\prime}}{d z}+4 H\left(\rho^{\prime}+p^{\prime}\right)= & \left(n_{\mathrm{SM}}^{\mathrm{eq}}(z)\right)^{2}\left\langle\sigma_{\mathrm{SM} \rightarrow \mathrm{DM}} v \Delta E\right\rangle \\
& +\left(n_{\mathrm{SM}}^{\mathrm{eq}}(z)\right)^{2}\left\langle\sigma_{\mathrm{SM} \rightarrow \gamma^{\prime}} v \Delta E\right\rangle .
\end{aligned}
$$

In reannihilation regime IIIa, the second term dominates the process of energy transfer. Plugging the equation of state $p^{\prime}\left(\rho^{\prime}\right)$ into this equation allows us to determine $\rho^{\prime}$, which in turns gives $T^{\prime}$ as the solution of

$$
\rho^{\prime}=\rho_{\gamma^{\prime}}^{\mathrm{eq}}\left(z^{\prime}\right)+\rho_{\mathrm{DM}}^{\mathrm{eq}}\left(z^{\prime}\right) .
$$

For instance, when $T^{\prime} \lesssim m_{\mathrm{DM}}$, the equation of state is $p^{\prime}=$ $\left(\rho^{\prime}-m_{\mathrm{DM}} Y_{\mathrm{DM}} s\right) / 3$, and $\rho_{\mathrm{DM}}\left(z^{\prime}\right)=\rho_{\mathrm{DM}}^{\mathrm{eq}}(z) Y_{\mathrm{DM}} / Y_{\mathrm{DM}}^{\mathrm{eq}}(z)$.

The DM production dynamics and the final amount of DM particles obtained from these Boltzmann equations along the reannihilation regime have been discussed in Ref. [2]. The final amount of DM it gives, $\Omega_{\mathrm{DM}}$, approximately scales as $\log \left(\left\langle\sigma_{\text {eff }} v\right\rangle\right) /\left\langle\sigma_{\gamma^{\prime} \rightarrow \mathrm{DM}} v\right\rangle$ (where $\left.\left\langle\sigma_{\mathrm{eff}} v\right\rangle \equiv \sqrt{\left\langle\sigma_{\mathrm{SM} \rightarrow \gamma^{\prime}} v\right\rangle\left\langle\sigma_{\mathrm{DM} \rightarrow \gamma^{\prime}} v\right\rangle}\right)$. This gives a $\Omega_{\mathrm{DM}} \propto$ $\log \left(\alpha^{\prime} \epsilon\right) / \alpha^{\prime 2}$ scaling, explaining why this regime leads to a line which is close to horizontal in the phase diagram of Fig. 4.

As said above, the IIIa reannihilation regime is a new regime that is absent in the case of a massless dark photon. In the latter case, the reannihilation regime which shows up is IIIb, along which the dark sector is populated through SM $\rightarrow$ DM processes; see Ref. [2]. In this case, the Boltzmann equations are the same as for the regime IIIa, trading the $\gamma_{\mathrm{SM} \gamma^{\prime}}$ source term in Eq. (3.22) for a $\gamma_{\mathrm{SM} \leftrightarrow \mathrm{DM}}$ source term in Eq. (3.23). For the massive dark photon case, we will see below that this regime occurs only when one includes the thermal corrections and when $m_{\gamma^{\prime}}$ is much smaller than $m_{\mathrm{DM}}$, so that the phase diagram is approximately the same as for the massless case (i.e., with the shape of a "mesa," displaying only Ia, IIIb, $\mathrm{IVb}, \mathrm{Va}$, and Vb regimes). As explained in Ref. [2], the regime IIIb leads to an abundance $\Omega_{\mathrm{DM}}$ which scales as $\log \left(\alpha^{\prime} \kappa\right) / \alpha^{\prime 2}$.

\section{Secluded freeze-out: Regimes IVa and IVb}

As we have seen, the reannihilation regimes occur when the slow source term producing hidden-sector particles from SM particles is still active at the time when the temperature of the thermalized hidden sector, $T^{\prime}$, becomes smaller than $m_{\mathrm{DM}}$. If the source term becomes inactive before $T^{\prime} \sim m_{\mathrm{DM}}$, the system would not be in the reannihilation regime, but instead, the DM particles would undergo a simple "secluded freeze-out." By this, we mean standard, textbook freeze-out, except that it takes place in a hidden sector, characterized by a temperature $T^{\prime}$ which differs from that of the visible sector, $T$ (see Ref. [2] for details). 
In practice, though, secluded freeze-out does not occur in the instances depicted in Figs. 4 and 5. This stems from the fact that, for the values of the masses considered for these figures, with in particular $m_{\gamma^{\prime}}<m_{\mathrm{DM}}$, the $\mathrm{SM} \rightarrow \gamma^{\prime}$ source term is still active at $T^{\prime} \sim m_{\mathrm{DM}}$. This is so because there is no IR mass scale which could cut off this source term at $T^{\prime} \gtrsim m_{\mathrm{DM}}$. Nevertheless, there are values of the masses for which the secluded freeze-out regime clearly occurs. This is in particular the case if $m_{\gamma^{\prime}}<m_{\mathrm{DM}}<m_{e}$, as in this case the mass of the electron cuts the $\mathrm{SM} \rightarrow \mathrm{DM}$ and $\mathrm{SM} \rightarrow \gamma^{\prime}$ source term at $T \sim m_{e}$, which can occur before $T^{\prime}$ goes below $m_{\mathrm{DM}}$. In principle, one can distinguish two regimes here, depending on whether the dominant source term comes from the $\mathrm{SM} \rightarrow \mathrm{DM}$ or $\mathrm{SM} \rightarrow \gamma^{\prime}$ processes, corresponding to the IVa and IVb regimes, respectively. However, in practice, without taking into account thermal corrections, in this case only the regime IVa takes place, because the $\mathrm{SM} \rightarrow \gamma^{\prime}$ source term naturally dominates in the region of parameter space in the secluded regime. Thermal corrections change this picture. As we shall see below, the regime IVb is the one which applies in this case. In all cases, the relic density is essentially determined by the value of $\alpha^{\prime}$, leading to an approximately horizontal line in the phase diagram; see Fig. 4 and Ref. [2].

\section{E. Freeze-out: Regimes Va and Vb}

Finally, from the reannihilation or secluded freeze-out regimes, if we increase $\kappa$ (and consequently $\epsilon$ ), at some point all particles form a single thermal bath, characterized by a single temperature $T$, so that DM undergoes a standard freeze-out. This happens when, on top of the $\alpha^{\prime}$-driven processes which were already in thermal equilibrium in the previous regimes, the $\kappa$-driven processes and/or the $\epsilon$-driven processes thermalize. Thus, such a transition into the freeze-out regime takes place when either $\kappa$ becomes larger than $\kappa_{\text {th }}$ [Eq. (3.8)] or $\epsilon$ becomes larger than $\epsilon_{\text {th }}$ [Eq. (3.10)]. Also, the freeze-out can be dominated either by the $\gamma^{\prime} \leftrightarrow \mathrm{DM}$ annihilation process (leading to regime $\mathrm{Va}$, depending only on the value of $\alpha^{\prime}$, the horizontal line in Fig. 6) or by the SM $\leftrightarrow$ DM process for larger values of $\kappa$ (leading to regime $\mathrm{Vb}$, which depends only on the value of $\kappa$, the vertical line in Fig. 6). The transition between regimes $\mathrm{Va}$ and $\mathrm{Vb}$ itself occurs when the $\mathrm{SM} \leftrightarrow \mathrm{DM}$ rate becomes larger than the $\gamma^{\prime} \leftrightarrow$ DM one.

\section{INCLUDING THERMAL EFFECTS}

All the discussion of DM production regimes above has been done without including any thermal effects and is generic of what could also happen in other models of DM based on three sectors with three connectors. The dark photon model we considered is a bit special, as it is known for displaying specific thermal effects, which can be important in some cases. This has been studied at length in the context of dark photon production, in particular in stars [28-33]. Based on these studies, we will discuss now these effects (focusing on dark photon production in the early Universe) and on the change they imply for each DM production regime unveiled in the previous section.

The most relevant issue concerns how to treat the limit $m_{\gamma^{\prime}} \rightarrow 0$, starting from a massive dark photon. Indeed, in the massive case, one distinguishes the dark photon interaction and mass eigenstate bases (see Sec. II above), while for $m_{\gamma^{\prime}} \rightarrow 0$, the $A_{\mu}$ and $A_{\mu}^{\prime}$ fields are degenerate, and the distinction between the bases disappears. More concretely, this implies that, in the massless limit, dark photons do not couple to SM particles and so cannot be produced; see the discussion following Eq. (2.2). How to take this effect properly into account is subtle, but in the presence of a medium, as in the Universe or inside stars, this has been extensively studied in the literature, and eventually clarified [28-33]. As this problem is central for a proper incorporation of the thermal effects, we recap the key results of these works in Appendix A, while here we only summarize the salient points, working in the interaction basis.

In a thermal bath, a propagating dark photon can mix with an ordinary photon, which in turn interacts with the plasma of charged particles. This process is depicted in Fig. 7. In this figure, the double (single) wiggly lines depict the dark photon (and, respectively, photon) propagators. The crossed circles represent their mixing $\propto \epsilon m_{\gamma^{\prime}}^{2}$, and the blob is the photon polarization in the thermal bath-for instance, made of relativistic $e^{+} e^{-}$particles. The dashed straight line is a cut associated with taking the imaginary part of the photon polarization tensor. In vacuum, if nonzero, this cut would be related to the decay rate of the dark photon into, say, $e^{+} e^{-}$pairs, but in a plasma it includes also the dark photon creation rate from coalescence processes, i.e., $e^{+} e^{-} \rightarrow \gamma^{\prime}$ [28,34]. By the same token, a cut in a two-loop diagram with photon exchange within the polarization tensor would lead to the dark photon creation rate from Compton scattering, say, $\gamma e^{ \pm} \rightarrow \gamma^{\prime} e^{ \pm}$, and pair annihilation, i.e., $e^{+} e^{-} \rightarrow \gamma \gamma^{\prime}$, etc.

On top of this, in a medium one must distinguish the behavior of the transverse and longitudinal components of the photon polarization tensor. The latter corresponds to genuine excitations of the medium, known as plasmons, see, e.g., Ref. [35]. This, in turn, impacts the creation rate for the production of transverse and longitudinal dark photons. As we discuss in Appendix A, in most of the

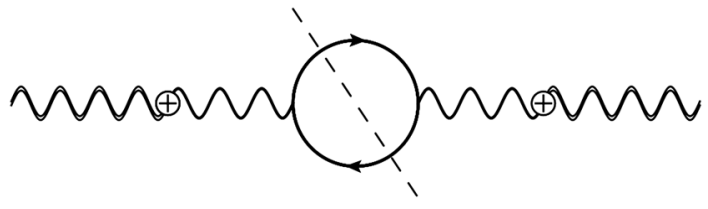

FIG. 7. The imaginary part of the dark photon propagator (double wiggly lines) in a medium includes both its decay rate and creation rates. 
range of interest for dark matter particle production in the early Universe, the creation of dark photons is dominantly through transverse photons [31,32]. In a thermal bath, the latter behave essentially as massive particles, with a thermal mass (here denoted as $\omega_{T}$ ) [36].

$$
\operatorname{Re} \Pi_{\gamma, T} \equiv \omega_{T}^{2} \sim \sum_{i} e_{i}^{2} T^{2},
$$

where $\Pi_{\gamma, T}$ is the self-energy of transverse photons and $e_{i}$ is the electric charge of the relativistic particles present in the primordial plasma. Taking this into account, the creation of transverse dark photons proceeds through an effective mixing parameter $\epsilon \rightarrow \epsilon_{\mathrm{eff}}$, with

$$
\epsilon \rightarrow \epsilon_{\mathrm{eff}}=\frac{\epsilon m_{\gamma^{\prime}}^{2}}{m_{\gamma^{\prime}}^{2}-\Pi_{\gamma, T}} .
$$

The numerator comes from the mixing mass term in Eq. (2.3), and the denominator from the transverse photon propagator with virtuality $k^{2}=m_{\gamma^{\prime}}^{2}$. In the resonance region $m_{\gamma^{\prime}} \approx \omega_{\gamma, T}$, one must take into account the finite width of in-medium transverse photon modes, $\propto \operatorname{Im} \Pi_{\gamma, T} \ll \operatorname{Re} \Pi_{\gamma, T}$. Production at the resonance $m_{\gamma^{\prime}} \sim \omega_{T}$ will play an important role in the sequel, but essentially, the substitution rule in Eq. (4.2) implies that the production of dark photons is suppressed at high temperature/low dark photon mass, $m_{\gamma^{\prime}} \ll \omega_{T}$. In particular, in the limit $m_{\gamma^{\prime}} \rightarrow 0, \epsilon_{\text {eff }} \rightarrow 0$, which means that there is no more SM $\leftrightarrow \gamma^{\prime}$ connection, so that the massless dark photon case is recovered smoothly (i.e., if $m_{\gamma^{\prime}}=0$, it can be rotated away and thus does not couple to SM EM currents). This phenomenon is familiar from the MSW effect in neutrino oscillations, in which oscillation into other neutrino flavors is suppressed by the frequent neutrino interactions in matter. In the opposite limit, however, $\epsilon_{\text {eff }} \rightarrow \epsilon$, and the production of dark photons is only suppressed by the bare mixing $\epsilon$ parameter, as in vacuum. ${ }^{9}$ Notice also that the substitution of Eq. (4.2) is for the production of (transverse) dark photons on the mass shell and not in processes with an exchange of virtual photons/dark photons, which are essentially insensitive to the dark photon mass. The rationale behind Eq. (4.2) is discussed in Appendix A.

The main practical consequences of the above discussion for the production of massive dark photons and DM in the early Universe are that dark photon production is suppressed at high temperatures (compared to its mass) and subsequently is resonantly enhanced once $\omega_{T} \approx m_{\gamma^{\prime}}$. The latter occurs at a temperature approximately equal to $T_{\text {res }} \simeq(\mathrm{a}$ few $) m_{\gamma^{\prime}}$. This implies that if $m_{\mathrm{DM}}$ is larger than $m_{\gamma^{\prime}}$ by more than 1 or 2 orders of magnitude-i.e., if

\footnotetext{
${ }^{9}$ The spontaneous symmetry breaking case, as compared to the Stückelberg one, involves additional subtleties discussed in Appendix B.
}

$m_{\mathrm{DM}} \gg T_{\text {res }}$ - there is basically no significant production of dark photons from the SM at times that are relevant for DM production, so one recovers a mesa-shaped phase diagram similar to the massless case. Thus, regimes Ib, II, IIIa, and IVa are irrelevant, and only regimes Ia, IIIb, IVb, $\mathrm{Va}$, and $\mathrm{Vb}$ remain, as in the massless case.

Figure 8 gives the evolution of the dark photon and DM yields, $Y_{\gamma^{\prime}}$ and $Y_{\mathrm{DM}}$, as a function of $m_{\gamma^{\prime}} / T$ and for two characteristic sets of parameters. The solid (dotted) lines show the evolution including (not including) the thermal corrections to dark photon production (see Appendix A for details). The dotted lines show the evolution of $Y_{\mathrm{DM}}$, taking $m_{\gamma^{\prime}}=0$ with other parameters unchanged.

In the first panel [Fig. 8(a)], we have taken $m_{\gamma^{\prime}}=1 \mathrm{GeV}$, $m_{\mathrm{DM}}=100 \mathrm{GeV}$, and couplings that lead to the observed relic density when we include the thermal effects $\left(\kappa=2 \times 10^{-11}\right.$ and $\left.\epsilon=5.6 \times 10^{-9}\right)$. Given that DM is 2 orders of magnitude heavier than the dark photon, this panel shows that, as expected, the production of dark photons is suppressed at high temperatures, and in particular at temperatures that are relevant for DM production, $T \gtrsim m_{\mathrm{DM}}$. As a result, when the dark photon yield becomes important, around $T_{\text {res }} \simeq m_{\gamma^{\prime}} /($ a few $)$, the production rate of DM from dark photon pair annihilation is already too much Boltzmann suppressed to play any sizeable role in the final amount of DM (red solid line). This means that DM is mostly produced directly through $\kappa$-driven $\mathrm{SM} \rightarrow \mathrm{DM}$ processes, and that the relic density is very close to the one obtained considering a massless dark photon (red dotted line). Thus, the value of $\kappa$ is close to the value one needs in the massless case, $\kappa_{\mathrm{II}} \simeq \kappa_{\mathrm{Ia}}=2 \times 10^{-11}$. If, instead, one considers the dark photon as massive but neglects the thermal corrections, there is no suppression of the dark photon production at high temperatures, and DM is sizeably produced through dark photons. As a result, one obtains a larger DM relic density (red dashed line). Therefore, without thermal corrections, one would need a smaller value of $\kappa$. Indeed, for these masses, regime II applies so that the overall scaling of the dark photon production of $\mathrm{DM}$ is $\propto \epsilon^{4} \alpha^{2} \propto \kappa^{4}$, in which case one would need $\kappa_{\text {II }}=2 \times 10^{-12}$.

The second panel [Fig. 8(b)] illustrates the opposite situation, where the dark photon and DM masses are closer, $m_{\gamma^{\prime}}=1 \mathrm{GeV}, m_{\mathrm{DM}}=3 \mathrm{GeV}$. Again, this is shown for values of the couplings that lead to the observed relic density taking into account thermal corrections, $\kappa_{\mathrm{II}}=3.6 \times$ $10^{-14}$ and $\epsilon=10^{-11}$. With thermal effects included, the resonance appears at a temperature close to $m_{\gamma^{\prime}} \sim m_{\mathrm{DM}}$. As the production rate of DM from dark photons is not Boltzmann suppressed when the dark photon production is resonantly enhanced, the DM abundance strongly increases, following the behavior of the dark photon abundance. Consequently, the thermal corrections strongly enhance the DM production from dark photon pair annihilation, and this occurs both with respect to the massive 


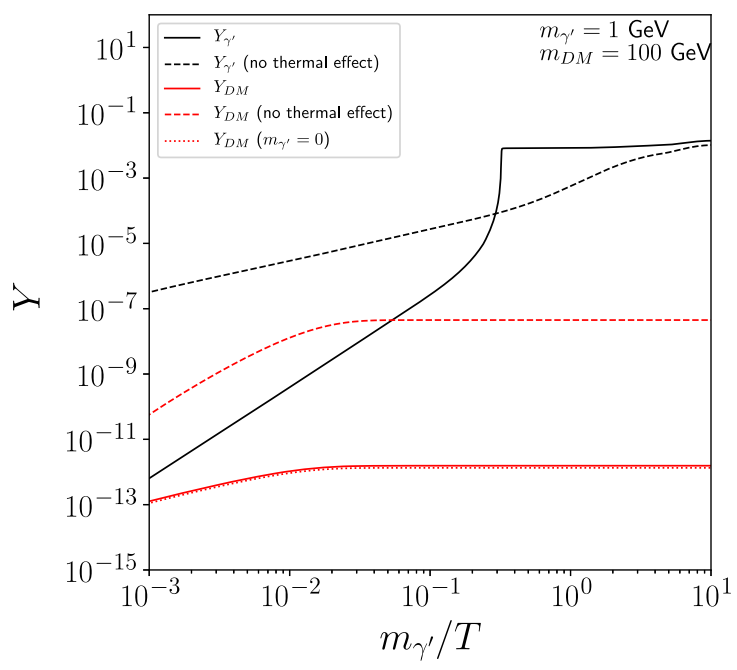

(a)

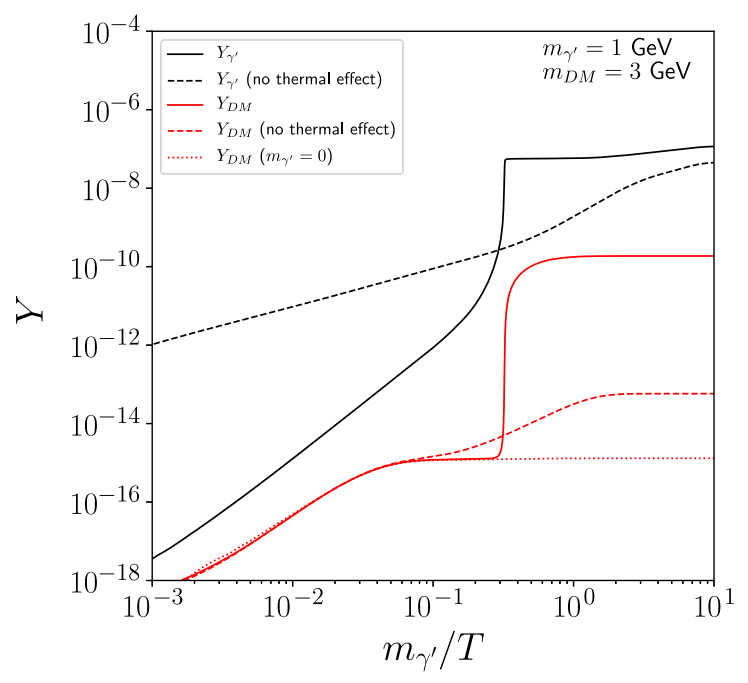

(b)

FIG. 8. Comparison of the evolution of the dark photon (black lines) and DM abundances (red lines), with (solid) and without (dashed) thermal effects on dark photon production. The dotted lines give the abundance of dark photons in the massless limit. Panels (a) and (b) differ by the choice of dark matter and dark photon masses. Both panels consider values of the couplings which lead to the observed relic density when the thermal corrections are taken into account (solid red lines): $\kappa=2 \times$ $10^{-11}$ and $\epsilon=5.6 \times 10^{-9}$ for panel (a), and $\kappa_{\mathrm{II}}=3.6 \times 10^{-14}$ and $\epsilon=10^{-11}$ for panel (b).

case without thermal correction and with respect to the massless case. This implies that the value of $\kappa$ needed with thermal corrections is smaller than the one required in the massless case, $\kappa_{\mathrm{Ia}} \approx 10^{-11}$, and is also smaller than the one needed in the massive case without thermal corrections, $\kappa_{\text {II }}=3 \times 10^{-13}$. In the latter case, the production is not suppressed at high temperatures but is not boosted afterwards by any resonance in the production. See Appendix A

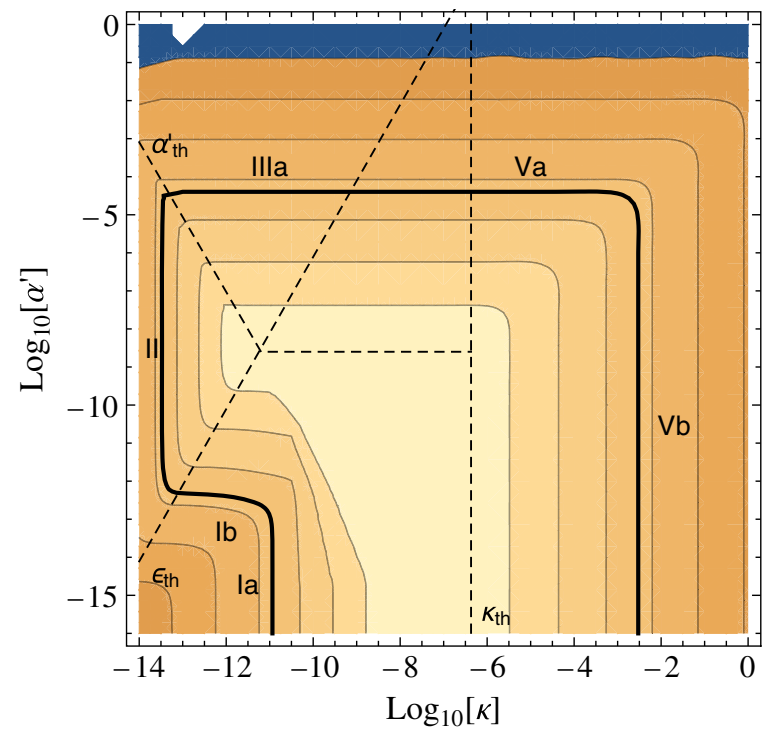

FIG. 9. Same as Fig. 4, with $m_{\mathrm{DM}}=3 \mathrm{GeV}$ and $m_{\gamma^{\prime}}=1 \mathrm{GeV}$, but taking into account thermal effects on dark photon production.

for a more detailed study of the effect of the resonance on the DM production.

One related general consequence of thermal effects is that they affect the thermalization of the dark photons with the SM thermal bath. As a result, the simple criterion $\Gamma / H>1$ of Eq. (3.9) has to be corrected, as the production can be resonantly enhanced around $T \sim T_{\text {res }}$ [see Eq. (4.2) and Appendix A]. Thus, taking into account thermal effects, in order to determine whether thermalization occurs [i.e., if $n_{\gamma^{\prime}}$ reaches $\sim n_{\gamma^{\prime}}^{\mathrm{eq}}(z)$ ], one needs to solve explicitly the Boltzmann equations. In this way, we find that the thermalization condition becomes

$$
\epsilon \gtrsim \epsilon_{\mathrm{th}} \equiv 6 \times 10^{-9}\left(\frac{m_{\mathrm{DM}}}{\mathrm{GeV}}\right)^{1 / 2}
$$

Comparing with Eq. (3.9), we see that, as expected, a smaller value of $\epsilon$ is needed for the dark photons to thermalize with the SM sector.

Figure 9 gives the phase diagram obtained including the thermal effects, for the same values of $m_{\mathrm{DM}}$ and $m_{\gamma^{\prime}}$ as in Fig. 4; i.e., $m_{\gamma^{\prime}}=1 \mathrm{GeV}, m_{\mathrm{DM}}=3 \mathrm{GeV}$. One observes that, as expected from Fig. 8, the "mooring bollard" shape of the phase diagram is accentuated by the thermal corrections, as the gap between the values of $\kappa$ in the Ia and II regimes is larger. Similarly, Fig. 10 gives the phase diagram including the thermal effects for the same values of the masses as in Fig. 5.

To explain the various changes in these phase diagrams, we now revisit each regime one by one.

Freeze-in regimes: In the Ia regime, the dark matter is produced directly by SM particles, and the dark photons 


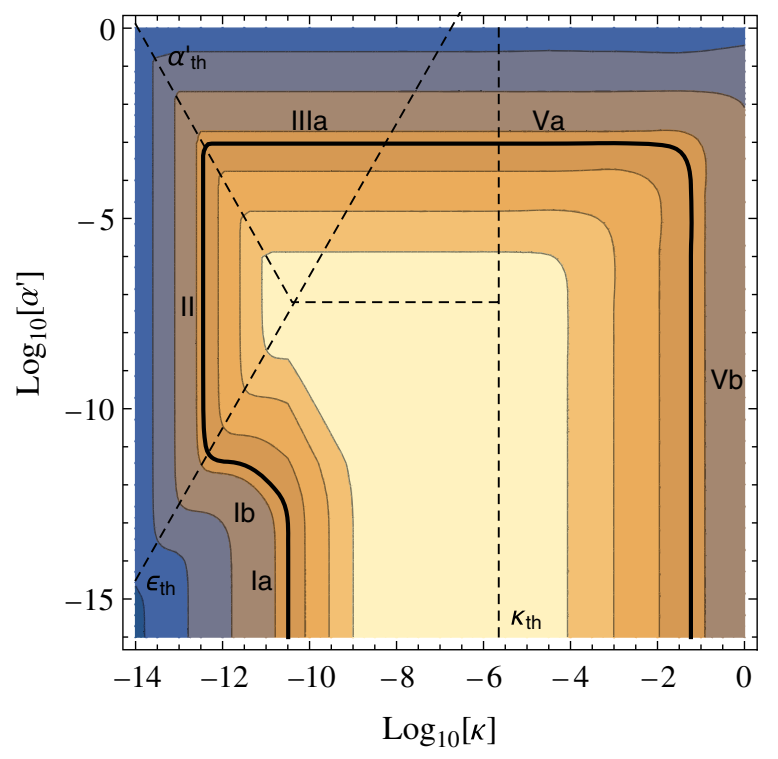

FIG. 10. Same as Fig. 5, with $m_{\mathrm{DM}}=100 \mathrm{GeV}$ and $m_{\gamma^{\prime}}=10 \mathrm{GeV}$, but with thermal effects included.

and their potential thermal corrections play no role in determining the dark matter abundance. ${ }^{10}$ In regime $\mathrm{Ib}$, instead, the approximation we used of taking $n_{\gamma^{\prime}}^{\text {eq }}(z)$ in the Boltzmann equation of Eq. (3.13) does not always give the correct result. Instead, one has to take the actual value of $n_{\gamma^{\prime}}$, as determined from Eq. (3.17). If $m_{\mathrm{DM}} \gg m_{\gamma^{\prime}}$, Eq. (3.17) will give $n_{\gamma^{\prime}} \approx 0$, and the $\mathrm{Ib}$ regime will disappear. Instead, if $m_{\mathrm{DM}} \gtrsim m_{\gamma^{\prime}}, n_{\gamma^{\prime}}$ will be enhanced by the resonance and regime Ib will be relevant for a larger range of couplings. The latter case is manifest in both phase diagrams with thermal effects. If the resonance is at $T<$ $m_{\mathrm{DM}} / 3$ (i.e., intermediate DM masses), the $\gamma^{\prime} \gamma^{\prime} \rightarrow$ DM DM freeze-in process is Boltzmann suppressed, and so it is not as efficient as assumed when deriving the $\alpha_{\mathrm{Ia}}^{\prime}$ coupling. Thus, the turn toward smaller values of $\kappa$ can happen for larger values of $\alpha^{\prime}$; see Fig. 10 for such an example.

Sequential freeze-in: For $m_{\gamma^{\prime}}$ not much smaller than $m_{\mathrm{DM}}$, as in Figs. 9 and 10, the thermal effects lead to smaller values of $\kappa$ than without thermal corrections (see the discussion on the second panel of Fig. 8, which gives the observed relic density along this regime). With thermal corrections, this regime leads to a vertical line in the phase diagram (as in the case without thermal corrections), because the $Y_{\gamma^{\prime}} \propto \epsilon^{2}$ and $\gamma_{\gamma^{\prime} \leftrightarrow \mathrm{DM}} \propto \alpha^{\prime 2}$ scalings remain unchanged. For $m_{\gamma^{\prime}} \ll m_{\mathrm{DM}}$, this regime and the intermediate regime $\mathrm{Ib}$ merge with the standard Ia freeze-in regime, as shown in Fig. 11 for two choices of dark photon masses (solid lines). Compared to Fig. 6 (reproduced as

\footnotetext{
${ }^{10}$ Notice, however, that plasmon decay can significantly influence the relic abundance of very light, sub-MeV, dark matter candidates [37].
}

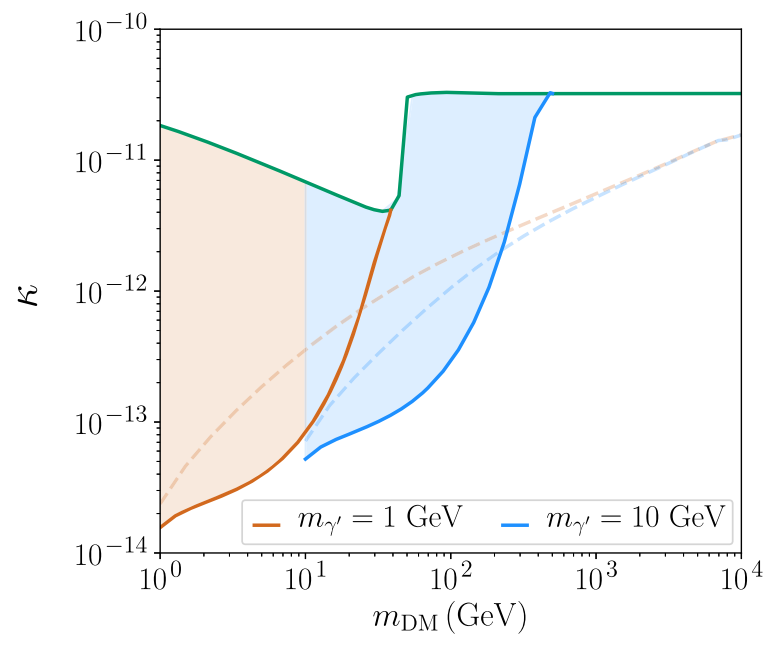

FIG. 11. Dark matter candidates as shown in Fig. 6, but with thermal corrections to dark photon production taken into account. The additional dashed lines are given for the sake of comparison. They correspond to the curves shown in Fig. 6, and thus are without thermal effects.

dashed curves), the noticeable features are that resonant production of dark photons leads to even smaller values of $\kappa$ (see the discussion revolving around Fig. 8) and that the regimes II (orange and blue curves) and Ia (green curve) merge for relatively smaller values of $m_{\mathrm{DM}} / m_{\gamma^{\prime}}$, for reasons explained above.

Reannihilation regimes: For $m_{\gamma^{\prime}}$ not much smaller than $m_{\mathrm{DM}}$, as in Figs. 9 and 10, we have seen above that the production of DM is boosted from the fact that the production of $\gamma^{\prime}$ is resonantly boosted at a temperature which is still relevant for DM production. Thus, the IIIa production regime-i.e., DM through $\gamma^{\prime}$-dominates even more over SM $\rightarrow$ DM production than in the case without thermal corrections (where it was already dominant; see above). Instead, for $m_{\gamma^{\prime}} \ll m_{\mathrm{DM}}$, regime IIIb, where SM $\rightarrow$ DM processes dominate, applies as in the massless case.

Secluded freeze-out: For $m_{\gamma}^{\prime}<m_{\mathrm{DM}}<m_{e}$, the IVa regime, which applies in the absence of thermal effects (see above), does not apply anymore, as in this case the production of dark photons is highly suppressed. This is due to the fact that, at $T \gg m_{\gamma^{\prime}}$, the $\gamma^{\prime}$ production is suppressed by thermal corrections and that for $T \sim m_{\gamma^{\prime}}<$ $m_{e}$ there is no sizeable resonant production, as the production is anyway Boltzmann suppressed. Regime IVb nevertheless can apply for $m_{\gamma^{\prime}} \ll m_{\mathrm{DM}}$, when production from the SM model is dominated by $Z$ decays, as in the massless case; see Ref. [2]. There is no IIIa-to-IVb transition (but only IIIa-to-Va transition) because, as $\kappa$ increases, so does $\epsilon$, and thus the production of dark photons increases as well.

Freeze-out regimes: Since all particles are thermalized in a single thermal bath, the effects of the above thermal corrections on the dynamics of these regimes are negligible. 


\section{COMMENTS ON CONSTRAINTS}

As the goal of our work is primarily the classification of the ways of producing DM, we only briefly comment on possible constraints, with a focus on the harder-to-test, small- $\kappa$ regimes. Indeed, as is well known, much of the parameter space corresponding to the freeze-out and reannihilation regimes of model (1.1) are already constrained or excluded, including by the direct-detection searches; see, e.g., Refs. [2,38]. For the parameter ranges relevant for the freeze-in regimes, no consequent production at colliders and indirect-detection experiments of hidden-sector particles is expected in general. The rates of processes relevant for those searches-i.e., $\mathrm{SM} \rightarrow \gamma^{\prime}$ or $\mathrm{SM} \rightarrow \gamma^{\prime} \rightarrow \mathrm{DM}$ processes-are typically strongly suppressed by the tiny parameters. Even so, if the mediator is light enough (meaning $m_{\gamma^{\prime}}<20 \mathrm{MeV}$ in the case of direct-detection searches of $\mathrm{DM}$ in the $m_{\mathrm{DM}} \gtrsim \mathrm{GeV}$ and above range), Rutherford scattering of DM on nuclei may be strongly enhanced, so that for some values of the masses, direct-detection experiments are already testing the freezein regime [38] (based on Refs. [2,39]). For lighter dark matter candidates, constraints on the parameter space of the freeze-in regime of millicharged dark matter will be possible in the future, exploiting the scattering of DM off electrons, provided the dark photon is light enough, $m_{\gamma^{\prime}} \lesssim$ $\mathrm{keV}$ [39]. Sequential freeze-in, as well as freeze-in regime $\mathrm{Ib}$, in which DM is produced by the mediator, here the dark photons extend the range of viable DM candidates toward even more feeble DM-to-SM couplings. In the case of the dark photon, the existence of these regimes is correlated to the mass of the dark photons, and in particular to the thermal effects discussed in Sec. IV. Specifically, for dark photons much lighter than DM, $m_{\gamma^{\prime}} \ll m_{\mathrm{DM}}$, the production of DM is effectively as in the massless dark photon regime, and so along the standard Ia freeze-in regime. This does not preclude the possibility that the new freeze-in regimes could also be tested by direct detection, including for models other than Eq. (1.1) [40].

Light dark photons and light millicharged particles are also constrained by stellar as well as CMB (cosmic microwave background) and BBN (big bang nucleosynthesis) measurements. In particular, the impact of massive, feebly coupled dark photons produced by freeze-in on cosmological observables has been studied in detail [41] (see also Ref. [33]). BBN can constrain dark photons that have an hadronic decay channel open, $m_{\gamma^{\prime}}>2 \times m_{\pi} \sim 300 \mathrm{MeV}$, while CMB measurements are relevant for lighter dark photons, $2 m_{e} \lesssim m_{\gamma^{\prime}} \lesssim 100 \mathrm{MeV}$, through their energy injection from dark photon decay into $e^{+} / e^{-}$. For instance, if $m_{\gamma^{\prime}}=1 \mathrm{GeV}$, as in the phase diagram of Fig. 9, then the range $10^{-12} \lesssim \epsilon \lesssim 10^{-10}$, which should show as an oblique band in the phase diagram of Fig. 4, is excluded by BBN, as can be seen from Fig. 5 of Ref. [41]. At the same time, for $m_{\mathrm{DM}}=3 \mathrm{GeV}$, the sequential freeze-in runs from roughly $10^{-12} \lesssim \epsilon_{\text {II }} \lesssim 3 \times 10^{-7}$, so that several candidates whose abundances are set by sequential freeze-in are not excluded by BBN measurements (or, for that matter, by any other kind of currently known constraints). For $m_{\gamma^{\prime}}=10 \mathrm{GeV}$, the range that is constrained by $\mathrm{BBN}$ corresponds to $5 \times$ $10^{-11} \lesssim \epsilon \lesssim 5 \times 10^{-12}$ and $10^{-14} \lesssim \epsilon \lesssim 10^{-13}$, while for $m_{\mathrm{DM}}=100 \mathrm{GeV}, 3 \times 10^{-12} \lesssim \epsilon_{\mathrm{II}} \lesssim 10^{-7}$, again leaving room for viable candidates in the sequential freeze-in regime.

\section{GENERALIZATION TO OTHER MODELS}

The model we considered above has a particular structure, where the three connections are determined by only two independent parameters. One could therefore wonder if the overall DM production regime picture we have obtained above is deeply connected to this particular structure or is more general. In this section, we would like to stress that this picture applies to other models and that in particular the sequential freeze-in regime could lead to the observed relic density in many setups. In some cases, it can even be the only possible way to produce DM.

To illustrate this discussion, let us take another example, where DM is a fermionic particle $\chi$, coupled to a scalar particle $\phi$ :

$$
\mathcal{L} \ni-\left(Y_{\chi} \bar{\chi} \chi \phi+\text { H.c. }\right)-\lambda_{\phi} \phi^{\dagger} \phi H^{\dagger} H .
$$

From such a structure, if the $\phi$ scalar field does not acquire any vacuum expectation value (VEV), clearly one cannot create any DM particle directly from the SM (at lowest order in $Y_{\chi}$ and $\lambda_{\phi}$ ). The way to produce DM from the SM is then to create first $\phi$ particles and from there DM particles. Thus, the three new regimes we presented above- Ib, II, IIIa, and IVa—are the generic possible ones, with $Y_{\chi}$ and $\lambda_{\phi}$ playing the roles of $e^{\prime}$ and $\epsilon$ in the kinetic mixing model above, respectively. In particular, if none of the interactions thermalize, the sequential freeze-in is the only possible one. If instead the $\phi$ scalar field has a VEV, it mixes with the Higgs boson, and one gets a structure very similar to that of the kinetic mixing model above, with two parameters inducing the three connections between the SM, $\phi$, and $\psi$ fluids. DM can be pair-produced from SM particles through Higgs and $\phi$ exchange, from a combination of the $Y_{\chi}$ and $\lambda_{\phi}$ interactions (similar to the $\kappa$ combination above), $\phi$ can be produced from the SM through $\lambda_{\phi}$ (similar to $\epsilon$ above), and DM particles can be produced by $\phi$ particles through the $Y_{\chi}$ interaction (similar to the $e^{\prime}$ coupling above). ${ }^{11}$ Thus, one expects a phase diagram similar to the one we obtained above.

The structure of the phase diagram of Fig. 4 is deeply connected to the fact that the sizes of the SM-to-mediator

\footnotetext{
${ }^{11}$ Note that, unless it is forbidden in various ways, we could also have a $\phi H^{\dagger} H$ interaction which implies a $\phi-h$ mixing too. In the presence of such a term, the various connections also depend on this interaction.
} 
and mediator-to-DM interactions imply a lower bound on the size of the processes which directly connect SM and DM particles. For the kinetic mixing case, this is quantified by the $\kappa=\epsilon \sqrt{\alpha^{\prime} / \alpha}$ relation. For the scalar mediation example of Eq. (6.1), a similar phenomena occurs. If instead one had three totally independent connections between the three sectors, still the various regimes encountered above would be possible, in particular the sequential freeze-in, but the overall picture would become more complicated, as the phase diagram becomes a 3D diagram, rather than a $2 \mathrm{D}$ one, as in Fig. $4 .{ }^{12}$ In this case, one can produce dark matter in the nine ways described above. Thus, at least nine regimes are possible, along the five different dynamical ways of producing DM from the SM, already mentioned above: freeze-in, sequential freeze-in, reannihilation, secluded freeze-out, and freeze-out.

\section{CONCLUSIONS}

In this work, we have analyzed the problem of DM production in the framework of a very popular model in which DM is charged under a hidden sector $U(1)^{\prime}$ and interacts with SM particles through kinetic mixing. It is impressive that such a simple framework can lead to a structure of DM production regimes as rich as the one we have uncovered. Indeed, we have found that DM production out of the SM thermal bath through the kinetic mixing portal involves no fewer than five different dynamical mechanisms, and this along nine distinct regimes. This result, which we obtained by considering the dark photon to be massive, generalizes the massless dark photon case for which four dynamical mechanisms, along five regimes, were operative [2]. The four new regimes, including the one based on a new DM production mechanism which we dub "sequential freeze-in" (regime II in our classification of possible regimes), all involve prior production of dark photons by SM particles. While we focused on the kinetic mixing portal model, the structure we have found is actually characteristic of DM setups in which, on top of the SM particles and DM particles, there are other particles, akin to the dark photons, which can couple both to the SM and to the DM particles. More precisely, it is characteristic of models in which these three fluids communicate along three connections, which are due to two different interactions, here $\alpha^{\prime}$ and $\epsilon$. Such a structure thus applies to many possible DM models. Despite the relative complexity of the DM production structure, a simple picture emerges in the

\footnotetext{
${ }^{12}$ A simple possibility of this kind would be to have a scalar mediator $\phi$ and a scalar DM particles $\phi_{\text {DM }}$ with three quartic terms, $\quad \mathcal{L} \ni-\lambda_{H-\phi} \phi^{\dagger} \phi H^{\dagger} H-\lambda_{\mathrm{DM}-H} \phi_{\mathrm{DM}}^{\dagger} \phi_{\mathrm{DM}} H^{\dagger} H-$ $\lambda_{\mathrm{DM}-\phi} \phi_{\mathrm{DM}}^{\dagger} \phi_{\mathrm{DM}} \phi^{\dagger} \phi$. If both $\phi$ and $\phi_{\mathrm{DM}}$ have no VEV, the three connections remain fully independent at tree level and lowest order in the couplings. Thus, one has still a minimum size for one connection as a function of the other two, but loop suppressed, which somewhat changes the overall picture.
}

phase diagram, as depicted in the plane $\alpha^{\prime}$ vs $\kappa=\epsilon \sqrt{\alpha^{\prime} / \alpha}$. Indeed, the different production regimes follow a universal pattern composed of vertical and (almost) horizontal lines in the phase diagrams, arranged in a shape suggesting a "mooring bollard."

In practice, the four new DM production regimes turn out to be operative when the mediator-here the dark photon-is lighter than the DM particles by a factor $m_{\gamma^{\prime}} / m_{\mathrm{DM}} \gtrsim 10^{-2}$. Below this bound, the mass of the hidden photon is effectively negligible, and one recovers the regimes of DM production of the massless case studied in detail in Ref. [2]. In particular, one recovers in this limit the characteristic "mesa" shape in the phase diagrams.

The new "sequential freeze-in" dynamical production mechanism (regime II) involves the slow, out-ofequilibrium production of dark photons, followed by a slow out-of-equilibrium production of DM from these dark photons. We find it interesting that such a chain of the freeze-in production of particles is operative and can lead to the observed relic density even in a model as simple as the kinetic portal. The other new regimes are DM freeze-in production from a thermalized population of dark photons (Ib), and reannihilation (IIIa) and secluded freeze-out (IVa) through the freeze-in production of dark photons. For these new regimes, the thermal effects on dark photon production turn out to be important. On the one hand, they suppress the production of dark photons, and thus of DM, at temperatures above the dark photon mass. On the other hand, they imply subsequently a resonant production of dark photons, which can strongly boost the DM production.

As a final comment, we stress that it is to be anticipated that, in more complicated models, involving more fluids and/or more independent connectors than considered here, the various possible regimes can be determined by following the strategy we followed in drawing Fig. 2. This strategy requires us first to determine the various possible connections between the fluids; second to look at the possibilities of combining these connections considering that each one either does reach the equilibrium before the DM number freezes or does not, but may still play a role by slowly producing one species from another one (along the freeze-in way); and finally to keep in mind that when a process in equilibrium decouples, the dynamics will also depend on whether the slow processes are still active.

\section{ACKNOWLEDGMENTS}

This work is supported by the F. R. S.-FNRS under Excellence of Science (EoS) Project No. 30820817, by the FRIA, by the "Probing dark matter with neutrinos" ULB-ARC convention, and by the IISN convention 4.4503.15. T. H. and L. V. thank the Erwin Schrödinger International Institute for hospitality while this work was completed. 


\section{APPENDIX A: THERMAL EFFECTS ON DARK PHOTON PRODUCTION}

The production rate of dark photons in a medium has been studied extensively-in particular in Refs. [28-33]. For comprehensiveness, we recap here the main steps of their arguments. We work in the interaction basis, treating mixing as a perturbation. Through mixing with SM photons, dark photons have a self-energy that inherits thermal features from those of photons (see Fig. 7),

$$
\Pi_{\gamma^{\prime}}=m_{\gamma^{\prime}}^{2}+\frac{\epsilon^{2} m_{\gamma^{\prime}}^{4}}{\left(K^{2}-\Pi_{\gamma}\right)},
$$

with $K^{2}=\omega^{2}-k^{2}$, where $K$ is the momentum of the virtual dark photon. In particular, its imaginary part is

$$
\operatorname{Im} \Pi_{\gamma^{\prime}}=\frac{\epsilon^{2} m_{\gamma^{\prime}}^{4} \operatorname{Im} \Pi_{\gamma}}{\left(m_{\gamma^{\prime}}^{2}-\operatorname{Re} \Pi_{\gamma}\right)^{2}+\operatorname{Im} \Pi_{\gamma}^{2}},
$$

where we set $K^{2}=m_{\gamma^{\prime}}^{2}$, corresponding to the mixing of a virtual photon into a dark photon on the mass shell. In vacuum, $\operatorname{Im}_{\gamma}$ is related to possible photon decay channels, but in a thermal bath, $\operatorname{Im} \Pi_{\gamma}$ has terms corresponding to both the emission and absorption rates of photons from the medium $^{13}$ :

$$
\operatorname{Im} \Pi_{\gamma}=-\omega \Gamma_{\gamma}=-\omega\left(\Gamma_{\gamma, \mathrm{em}}-\Gamma_{\gamma, \mathrm{abs}}\right) .
$$

By detailed balance, ${ }^{14}$ the emission rate of a photon of energy $\omega$ is Boltzmann suppressed compared to the corresponding absorption rate,

$$
\Gamma_{\mathrm{em}}=\exp (-\omega / T) \Gamma_{\mathrm{abs}}
$$

so that

$$
\Gamma_{\gamma^{\prime}, \mathrm{em}}=\frac{\epsilon^{2} m_{\gamma^{\prime}}^{4} \Gamma_{\gamma, \mathrm{em}}}{\left(m_{\gamma^{\prime}}^{2}-\operatorname{Re} \Pi_{\gamma}\right)^{2}+\omega^{2}\left(e^{\omega / T}-1\right)^{2} \Gamma_{\gamma, \mathrm{em}}^{2}} .
$$

Now, at finite temperature, on top of genuine transverse photons, the medium can also sustain the propagation of

\footnotetext{
${ }^{13}$ More precisely, $\Gamma$ is to be interpreted as the rate that determines the approach to thermal equilibrium,

$$
f(\omega, t)-f_{\text {eq }} \propto \exp (-\Gamma t),
$$

where $f$ is the distribution function of (here) dark photons. The strange minus sign between the rates of emission and of absorption can be traced to Bose-Einstein statistics; for fermions, $\Gamma=\Gamma_{\mathrm{em}}+\Gamma_{\mathrm{abs}}[34]$

${ }^{14}$ More generally, this is due to the fact that the amplitude squared for the emission and absorption process are equal, due to unitarity [42].
}

longitudinal modes or plasmon waves. These correspond to oscillations of charged particles that composed the thermal bath. Thus, in the expression of the rate of production of dark photons given by Eq. (A.5), one must thus distinguish transverse $(T)$ and longitudinal $(L)$ self-energy, and therefore emission/absorption rates, $(\Gamma, \Pi,) \rightarrow\left(\Gamma_{T, L}, \Pi_{T, L}\right)[31]$.

The transverse modes, corresponding to ordinary photons dressed by interaction with the medium, are the simplest. In the relativistic regime and to leading order in $\alpha$, the transverse photons simply get a thermal mass, albeit with a slight momentum dependence,

$$
\operatorname{Re}_{\gamma, T}=\left\{\begin{array}{ll}
\omega_{P}^{2}=\sum_{i} q_{i}^{2} T^{2} / 9 & \text { low } k \\
3 / 2 \omega_{P}^{2} & \text { large } k
\end{array},\right.
$$

where $\omega_{P}$ is the so-called plasma frequency and the sum is over relativistic charged particles [36]. For later use, we mention the fact that there is no substantial wave function renormalization for the transverse modes.

The longitudinal mode self-energy has a more involved structure. This is related to the fact that longitudinal photons do not propagate in vacuum. Still in the relativistic regime and to leading order in $\alpha$, the self-energy takes the form $^{15}[36]$

$$
\Pi_{L}(\omega, k)=3 \omega_{P}^{2} \frac{K^{2}}{k^{2}}\left(\frac{\omega}{2 k} \log \left(\frac{\omega+k}{\omega-k}\right)-1\right) .
$$

Solving for $\omega_{L}^{2}-k^{2}=\operatorname{Re}_{L}\left(\omega_{L}(k), k\right)$ leads to

$$
\operatorname{Re} \Pi_{\gamma, L}=\left\{\begin{array}{ll}
\omega_{P}^{2} K^{2} / \omega^{2} & k \sim 0 \\
\sim 0 & k \gtrsim \omega_{P}
\end{array} .\right.
$$

The behaviors of the dispersion relations of the transverse (solid blue) and longitudinal (solid orange) modes are depicted in Fig. 12.

For a longitudinal plasmon mode close to on shell, $\omega \sim \omega_{L}, \quad \operatorname{Re} \Pi_{\gamma, L} \approx \omega_{L}^{2} K^{2} / \omega^{2}, \quad$ and one can write $^{16}$ $[31,32,36]$

$$
\frac{1}{K^{2}-\Pi_{L}} \approx \frac{\omega^{2} Z_{L}}{K^{2}\left(\omega^{2}-\omega_{L}^{2}\right)-i Z_{L} \omega^{2} \operatorname{Im} \Pi_{L}},
$$

where, using Eq. (A.7), the wave-function normalization $Z_{L}$ is given by

\footnotetext{
${ }^{15}$ We use here the definition of the longitudinal polarization tensor of Refs. [31,32], $\Pi_{L} \equiv \Pi_{L}^{\mathrm{APP}}$, which differs from that of Ref. [36], $\Pi_{L}^{\mathrm{BS}}=K^{2} / k^{2} \Pi_{L}^{\mathrm{APP}}$.

${ }^{16}$ To be clear, while we consider the real part of the self-energy only to leading order in $\alpha$, the imaginary part $\operatorname{Im} \Pi_{L}$ may include higher-order corrections in $\alpha$, so as to describe, for instance, Compton emission, etc.
} 


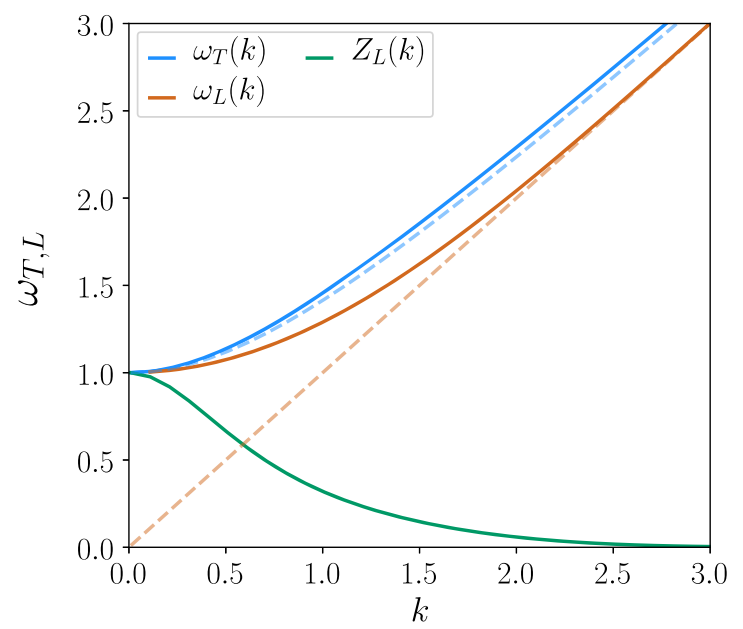

FIG. 12. Dispersion relations for $\omega_{T}(k)$ (blue solid line) and $\omega_{L}(k)$ (orange solid line) in the relativistic regime, $T \gg m_{e}$. They are normalized to $\omega_{P}=1$. The dashed orange line is the dispersion relation of a standard massive particle. The dispersion relation of the transverse mode has $\omega_{T}=\omega_{P}$ for small $k$ and $\omega_{T} \approx \sqrt{3 / 2 \omega_{P}^{2}+k^{2}}$ for large $k$. That of the longitudinal mode has $\omega_{L}=\omega_{P}$ for small $k$ but asymptotes to $\omega_{L} \approx k$ for large $k$. Also shown is the wave-function normalization $Z_{L}$ (green solid line) which goes to zero at large $k$, revealing that the longitudinal mode propagates only for small enough momenta.

$$
Z_{L}^{-1}=1+\frac{k^{2}}{\omega_{L}} \frac{3 \omega_{P}^{2}-\omega_{L}^{2}+k^{2}}{2\left(\omega_{L}^{2}-k^{2}\right)}
$$

which satisfies $Z_{L} \rightarrow 1$ as $k \rightarrow 0$ but goes to zero for $k \gtrsim \omega_{P}$, as shown in Fig. 12 (green solid line). This reflects the fact that the longitudinal mode mostly exists for moderate momenta. From this and Eq. (A.5), we get $^{17}$

$$
\Gamma_{\gamma^{\prime}, \mathrm{em}}^{L}=\frac{\epsilon^{2} m_{\gamma^{\prime}}^{4} \tilde{Z}_{L}^{2} \Gamma_{\gamma, \mathrm{em}}^{L}}{\left(\omega^{2}-\omega_{L}^{2}\right)^{2}+\omega^{2}\left(e^{\omega / T}-1\right)^{2}\left(\tilde{Z}_{L} \Gamma_{\gamma, \mathrm{em}}^{L}\right)^{2}},
$$

with $\tilde{Z}_{L}=\omega^{2} / m_{\gamma^{\prime}}^{2} Z_{L}$. This is to be compared to

$$
\Gamma_{\gamma^{\prime}, \mathrm{em}}^{T}=\frac{\epsilon^{2} m_{\gamma^{\prime}}^{4} \Gamma_{\gamma, \mathrm{em}}^{T}}{\left(m_{\gamma^{\prime}}^{2}-\omega_{T}^{2}\right)^{2}+\omega^{2}\left(e^{\omega / T}-1\right)^{2}\left(\Gamma_{\gamma, \mathrm{em}}^{T}\right)^{2}} .
$$

Comparing these expressions, one sees that at large $T$, $\omega_{P} \gg m_{\gamma^{\prime}}$, and $\omega \sim \omega_{P}$,

\footnotetext{
${ }^{17}$ Expression (A.11) agrees with the relevant literature [31,32], see also Ref. [43], but perhaps a comment is in order. For instance, it may be compared with Eq. (2.7) in Ref. [32] using the fact that in Eq. (A.11) the longitudinal photon emission rates are to be calculated as in vacuum, while in Ref. [32] (abbreviated RR) they include factors interpreted as wave-function normalization: $\left.\tilde{Z}_{L} \Gamma_{\gamma, \mathrm{em}}^{L}\right|_{\mathrm{us}}=\left.\Gamma_{\gamma, \mathrm{em}}^{L}\right|_{\mathrm{RR}}$.
}

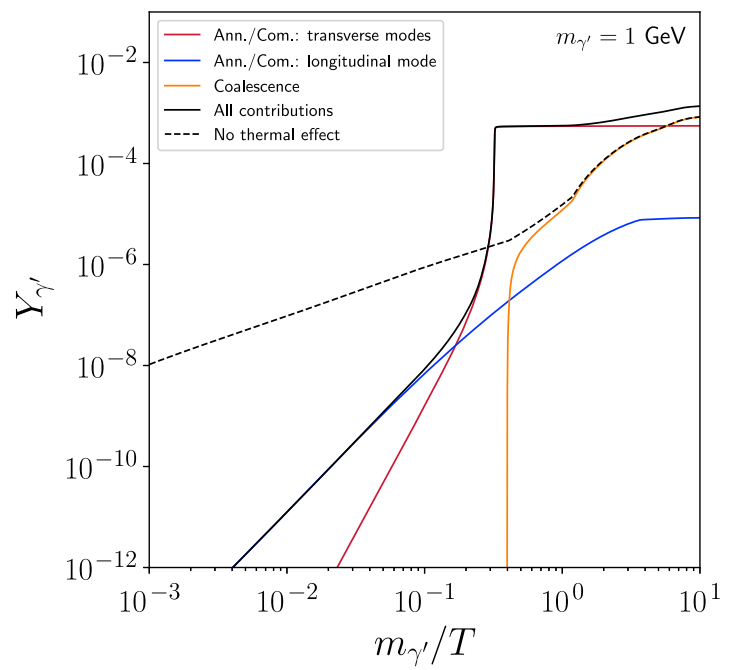

FIG. 13. For $\epsilon=10^{-9}$, all contributions to the dark photon yield as a function of the inverse temperature. One can distinguish contributions from pair annihilation and Compton processes for both transverse (red dashed line) and longitudinal modes (blue dashed line), from the coalescence process (orange dashed line) and from all contributions together (solid black line). For the coalescence, we have taken into account the thermal corrections to the mass of the SM particles that annihilate into a dark photon [30].

$$
\frac{\Gamma_{\gamma^{\prime}, \mathrm{em}}^{L}}{\Gamma_{\gamma^{\prime}, \mathrm{em}}^{T}} \approx \frac{\omega^{4}}{m_{\gamma^{\prime}}^{4}} \frac{\Gamma_{\gamma, \mathrm{em}}^{L}}{\Gamma_{\gamma, \mathrm{em}}^{T}} .
$$

As the rate for production of longitudinal photon modes itself is $\propto m_{\gamma^{\prime}}^{2}$, due to current conservation, $k_{\mu} J^{\mu}=0 \rightarrow$ $\epsilon_{\mu}^{L} J^{\mu} \propto m_{\gamma^{\prime}}$, the overall scaling of this ratio is $\omega^{2} / m_{\gamma^{\prime}}^{2} \gg 1$. So the production of dark photons is dominantly through the production of longitudinal photons at high temperature, $\omega_{P} \gtrsim m_{\gamma^{\prime}}[31,32]$. At lower temperatures, however, which is the regime relevant for infrared dominated freeze-in production,

$$
\frac{\Gamma_{\gamma^{\prime}, \mathrm{em}}^{L}}{\Gamma_{\gamma^{\prime}, \mathrm{em}}^{T}} \approx Z_{L}^{2} \frac{\Gamma_{\gamma, \mathrm{em}}^{L}}{\Gamma_{\gamma, \mathrm{em}}^{T}} \sim Z_{L}^{2} \frac{m_{\gamma^{\prime}}}{\omega} \ll 1,
$$

as $Z_{L} \gtrsim 1$, so production is dominantly through bona fide transverse photons [31,32]. Thus, the production of onshell dark photons proceeds essentially as in vacuum, but with a cross section in which the mixing parameter should be replaced by

$$
\epsilon \rightarrow \epsilon_{\mathrm{eff}}^{2}=\frac{\epsilon^{2} m_{\gamma^{\prime}}^{4}}{\left(m_{\gamma^{\prime}}^{2}-\operatorname{Re} \Pi_{\gamma}\right)^{2}+\omega^{2}\left(e^{\omega / T}-1\right)^{2} \Gamma_{\gamma}^{2}},
$$

legitimating the rule of thumb stated in Eq. (4.2).

Figure 13 illustrates all the contributions to the production of dark photons. There, one recognizes the hightemperature suppression of transversal modes [Eq. (A.13)] 
and the low-temperature suppression of the longitudinal mode [Eq. (A.14)].

\section{APPENDIX B: A NOTE ON DARK HIGGS PRODUCTION}

In the body of this work, we assumed that the only relevant d.o.f. for DM production are, besides the SM particles, the dark photon and the dark matter itself. They thus apply as such if the mass of the dark photon arises through the Stückelberg mechanism. If, instead, the $U(1)^{\prime}$ is broken through the Brout-Englert-Higgs mechanism, our underlying basic assumption is that the dark Higgs $\left(h^{\prime}\right)$ is much heavier than both the dark photon and the dark matter; i.e., that

$$
\frac{m_{h^{\prime}}}{m_{\gamma^{\prime}}}=\sqrt{\frac{\lambda^{\prime}}{2 \pi \alpha^{\prime}}} \gg 1 \quad \text { and } \quad \frac{m_{h^{\prime}}}{m_{\mathrm{DM}}}=\frac{\sqrt{2 \lambda^{\prime}} v^{\prime}}{m_{\mathrm{DM}}} \gg 1
$$

where $\lambda^{\prime}$ and $v^{\prime}$ are the dark Higgs quartic coupling and VEV. We assumed, for simplicity, that the DM is vectorlike so that $m_{\mathrm{DM}}$ can be taken to be independent of $v^{\prime}$, a hypothesis that could be relaxed in a more elaborate scenario. Now $\alpha^{\prime}$ is a (very) tiny parameter for most of the parameter space we consider, so these conditions can be easily met. If this is not the case (for instance, if for some reason $\lambda^{\prime}$ itself is a small parameter), then we may have, e.g.,

$$
m_{h^{\prime}} \sim m_{\gamma^{\prime}} \ll m_{\mathrm{DM}} .
$$

If this is the case, the system becomes more complex, as we must in principle take into account the abundance of $h^{\prime}$ particles on top of that of the DM and the dark photons; see Fig. 14.

An interesting aspect of this scenario is that the process of associated production of dark Higgs (or darkHiggsstrahlung) SM $\rightarrow h^{\prime} \gamma^{\prime}$ is unsuppressed in the limit $m_{\gamma^{\prime}} \rightarrow 0$, and scales as $[44,45]$

$$
\sigma_{\mathrm{SM} \rightarrow h^{\prime} \gamma^{\prime}} \propto \frac{\alpha^{2} \kappa^{2}}{s}
$$

for large $\sqrt{s} \gg\left(m_{h^{\prime}}+m_{\gamma^{\prime}}\right)$. As $\kappa=\epsilon \sqrt{\alpha^{\prime} / \alpha}$, the rate of $h^{\prime}$ production is proportional to $\alpha^{\prime}$ and not $\alpha^{\prime 2}$ as naively expected from the vertex $\mathcal{L} \supset e^{\prime 2} v^{\prime} h^{\prime} A_{\mu}^{\prime} A^{\prime \mu} \equiv$ $e^{\prime} m_{\gamma^{\prime}} h^{\prime} A_{\mu}^{\prime} A^{\prime \mu}$. This result, familiar from SM Higgs physics,

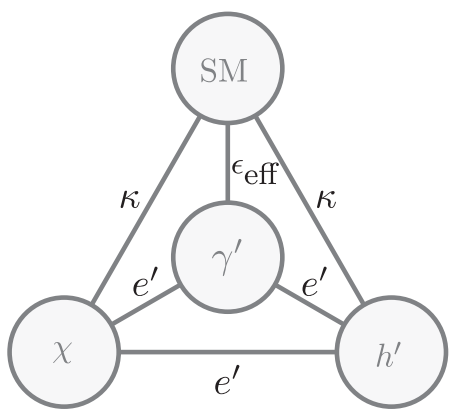

FIG. 14. The sectors and their connections in the Higgs phase of the dark photon model.

can be traced back to the fact that the process (B.3) is dominated by longitudinal $\gamma^{\prime}$ emission at large energies. Hence, the connection between the SM sector and the $h^{\prime}$ is controlled by $\kappa$, precisely like the DM itself; see Fig. 14. By the same token, the connections between the $h^{\prime}$, the $\gamma^{\prime}$, and the DM are all driven by $e^{\prime}$.

From the above discussion and the (approximate) symmetry of Fig. 14, we thus expect that the production of the dark Higgs will proceed as that of DM, starting from the freeze-in regime Ia for tiny values of $\alpha^{\prime}$, then $\mathrm{Ib}$ with the production of $h^{\prime}$ and DM from $\gamma^{\prime}$ in thermal equilibrium with the SM sector, etc. So the abundance of $h^{\prime}$ should essentially track that of DM, $Y_{h^{\prime}} \sim Y_{\mathrm{DM}}$, and this at least as long as $T \gtrsim m_{\mathrm{DM}}$. Thus, if sequential freeze-in occurs, the contribution of the process $h^{\prime} \gamma^{\prime} \rightarrow \chi \bar{\chi}$ should be subdominant compared to that of the process $\gamma^{\prime} \gamma^{\prime} \rightarrow \chi \bar{\chi}$. Consequently, and while a more detailed analysis may be of interest, we tentatively conclude that, generically speaking, the presence of $h^{\prime}$ should not affect significantly the structure of the phase diagram depicted in Figs. 9 and 10.

As for the symmetric phase, in which $m_{\gamma^{\prime}}=0$, it would contain two DM candidates, as the complex scalar associated with the dark Higgs would also be stable (by charge conservation), and both would be produced at similar rates for tiny couplings. In particular, as the abundance at freeze-in is $Y_{\mathrm{DM}} \propto 1 / m_{\mathrm{DM}}$ (barring production from $Z$-boson decay; see Ref. [2]), both particles will give (roughly) a similar contribution to the DM energy density, $\Omega_{\mathrm{DM}} \propto m_{\mathrm{DM}} Y_{\mathrm{DM}}$. While reannihilation and subsequent regimes may have a richer structure than in a scheme with a single DM candidate, we expect the phase diagram to follow essentially the "mesa" pattern studied in Ref. [2]. 
[1] B. Patt and F. Wilczek, Higgs-field portal into hidden sectors, arXiv:hep-ph/0605188.

[2] X. Chu, T. Hambye, and M. H. G. Tytgat, The four basic ways of creating dark matter through a portal, J. Cosmol. Astropart. Phys. 05 (2012) 034.

[3] J. Jaeckel and A. Ringwald, The low-energy frontier of particle physics, Annu. Rev. Nucl. Part. Sci. 60, 405 (2010).

[4] R. Essig et al., Working group report: New light weakly coupled particles, in Proceedings, 2013 Community Summer Study on the Future of U.S. Particle Physics: Snowmass on the Mississippi (CSS2013): Minneapolis, MN, USA, 2013 (2013), http://inspirehep.net/record/1263039?ln=en.

[5] J. Alexander et al., Dark sectors 2016 workshop: Community report, arXiv:1608.08632.

[6] R. Foot and S. Vagnozzi, Dissipative hidden sector dark matter, Phys. Rev. D 91, 023512 (2015).

[7] L. Ackerman, M. R. Buckley, S. M. Carroll, and M. Kamionkowski, Dark matter and dark radiation, Phys. Rev. D 79, 023519 (2009).

[8] J. L. Feng, H. Tu, and H.-B. Yu, Thermal relics in hidden sectors, J. Cosmol. Astropart. Phys. 10 (2008) 043.

[9] J. L. Feng, M. Kaplinghat, H. Tu, and H.-B. Yu, Hidden charged dark matter, J. Cosmol. Astropart. Phys. 07 (2009) 004.

[10] T. Hambye, On the stability of particle dark matter, Proc. Sci., IDM2010 (2011) 098 [arXiv:1012.4587].

[11] B. Holdom, Two U(1)'s and epsilon charge shifts, Phys. Lett. 166B, 196 (1986).

[12] E. C. G. Stueckelberg, Interaction energy in electrodynamics and in the field theory of nuclear forces, Helv. Phys. Acta 11, 225 (1938).

[13] F. Englert and R. Brout, Broken Symmetry and the Mass of Gauge Vector Mesons, Phys. Rev. Lett. 13, 321 (1964).

[14] P. W. Higgs, Broken Symmetries and the Masses of Gauge Bosons, Phys. Rev. Lett. 13, 508 (1964).

[15] J. McDonald, Thermally Generated Gauge Singlet Scalars as Self-Interacting Dark Matter, Phys. Rev. Lett. 88, 091304 (2002).

[16] L. J. Hall, K. Jedamzik, J. March-Russell, and S. M. West, Freeze-in production of FIMP dark matter, J. High Energy Phys. 03 (2010) 080.

[17] N. Bernal, M. Heikinheimo, T. Tenkanen, K. Tuominen, and V. Vaskonen, The dawn of FIMP dark matter: A review of models and constraints, Int. J. Mod. Phys. A 32, 1730023 (2017).

[18] M. Pospelov, A. Ritz, and M. B. Voloshin, Secluded WIMP dark matter, Phys. Lett. B 662, 53 (2008).

[19] M. Pospelov, Secluded $U(1)$ below the weak scale, Phys. Rev. D 80, 095002 (2009).

[20] R. Foot, Experimental signatures of a massive mirror photon, arXiv:hep-ph/9407331.

[21] P. Gondolo and G. Gelmini, Cosmic abundances of stable particles: Improved analysis, Nucl. Phys. B360, 145 (1991).

[22] A. Merle, V. Niro, and D. Schmidt, New production mechanism for $\mathrm{keV}$ sterile neutrino dark matter by decays of frozen-in scalars, J. Cosmol. Astropart. Phys. 03 (2014) 028.

[23] J. Heeck and D. Teresi, Cold keV dark matter from decays and scatterings, Phys. Rev. D 96, 035018 (2017).
[24] S. Koren and R. McGehee, Freeze-twin dark matter, arXiv: 1908.03559.

[25] G. L. Kane, P. Kumar, B. D. Nelson, and B. Zheng, Dark matter production mechanisms with a nonthermal cosmological history: A classification, Phys. Rev. D 93, 063527 (2016).

[26] M. Klasen and C. E. Yaguna, Warm and cold fermionic dark matter via freeze-in, J. Cosmol. Astropart. Phys. 11 (2013) 039.

[27] S. Heeba and F. Kahlhoefer, Probing the freeze-in mechanism in dark matter models with $U(1)^{\prime}$ gauge extensions, arXiv: 1908.09834.

[28] J. Redondo, Helioscope bounds on hidden sector photons, J. Cosmol. Astropart. Phys. 07 (2008) 008.

[29] J. Jaeckel, J. Redondo, and A. Ringwald, Signatures of a Hidden Cosmic Microwave Background, Phys. Rev. Lett. 101, 131801 (2008).

[30] J. Redondo and M. Postma, Massive hidden photons as lukewarm dark matter, J. Cosmol. Astropart. Phys. 02 (2009) 005.

[31] H. An, M. Pospelov, and J. Pradler, New stellar constraints on dark photons, Phys. Lett. B 725, 190 (2013).

[32] J. Redondo and G. Raffelt, Solar constraints on hidden photons re-visited, J. Cosmol. Astropart. Phys. 08 (2013) 034.

[33] A. Fradette, M. Pospelov, J. Pradler, and A. Ritz, Cosmological constraints on very dark photons, Phys. Rev. D 90, 035022 (2014).

[34] H. A. Weldon, Simple rules for discontinuities in finite temperature field theory, Phys. Rev. D 28, 2007 (1983).

[35] M. L. Bellac, Thermal field theory, in Cambridge Monographs on Mathematical Physics (Cambridge University Press, Cambridge, England, 2011).

[36] E. Braaten and D. Segel, Neutrino energy loss from the plasma process at all temperatures and densities, Phys. Rev. D 48, 1478 (1993).

[37] C. Dvorkin, T. Lin, and K. Schutz, Making dark matter out of light: Freeze-in from plasma effects, Phys. Rev. D 99, 115009 (2019).

[38] T. Hambye, M. H. G. Tytgat, J. Vandecasteele, and L. Vanderheyden, Dark matter direct detection is testing freeze-in, Phys. Rev. D 98, 075017 (2018).

[39] R. Essig, J. Mardon, and T. Volansky, Direct detection of sub-GeV dark matter, Phys. Rev. D 85, 076007 (2012).

[40] T. Hambye, M. H. G. Tytgat, J. Vandecasteele, and L. Vanderheyden (to be published).

[41] J. Berger, K. Jedamzik, and D. G. E. Walker, Cosmological constraints on decoupled dark photons and dark higgs, J. Cosmol. Astropart. Phys. 11 (2016) 032.

[42] S. Weinberg, Cosmological Production of Baryons, Phys. Rev. Lett. 42, 850 (1979).

[43] E. Rrapaj, A. Sieverding, and Y.-Z. Qian, Rate of dark photon emission from electron positron annihilation in massive stars, Phys. Rev. D 100, 023009 (2019).

[44] M. Pospelov, A. Ritz, and M. B. Voloshin, Bosonic superWIMPs as keV-scale dark matter, Phys. Rev. D 78, 115012 (2008).

[45] B. Batell, M. Pospelov, and A. Ritz, Probing a secluded $\mathrm{U}(1)$ at $B$-factories, Phys. Rev. D 79, 115008 (2009). 